\title{
Low-Profile UHF Antenna Design Based on an Anisotropic Transverse Resonance Condition
}

\author{
by Gregory Mitchell and Wasyl Wasylkiwskyj
}




\section{NOTICES}

\section{Disclaimers}

The findings in this report are not to be construed as an official Department of the Army position unless so designated by other authorized documents.

Citation of manufacturer's or trade names does not constitute an official endorsement or approval of the use thereof.

Destroy this report when it is no longer needed. Do not return it to the originator. 


\section{Army Research Laboratory}

Adelphi, MD 20783-1138

ARL-TR-7035

August 2014

\section{Low-Profile UHF Antenna Design Based on an Anisotropic Transverse Resonance Condition}

Gregory Mitchell and Wasyl Wasylkiwskyj

Sensors and Electron Devices Directorate, ARL 


\section{REPORT DOCUMENTATION PAGE}

Form Approved OMB No. 0704-0188

Public reporting burden for this collection of information is estimated to average 1 hour per response, including the time for reviewing instructions, searching existing data sources, gathering and maintaining the data needed, and completing and reviewing the collection information. Send comments regarding this burden estimate or any other aspect of this collection of information, including suggestions for reducing the burden, to Department of Defense, Washington Headquarters Services, Directorate for Information Operations and Reports (0704-0188), 1215 Jefferson Davis Highway, Suite 1204, Arlington, VA 22202-4302. Respondents should be aware that notwithstanding any other provision of law, no person shall be subject to any penalty for failing to comply with a collection of information if it does not display a currently valid OMB control number

PLEASE DO NOT RETURN YOUR FORM TO THE ABOVE ADDRESS.

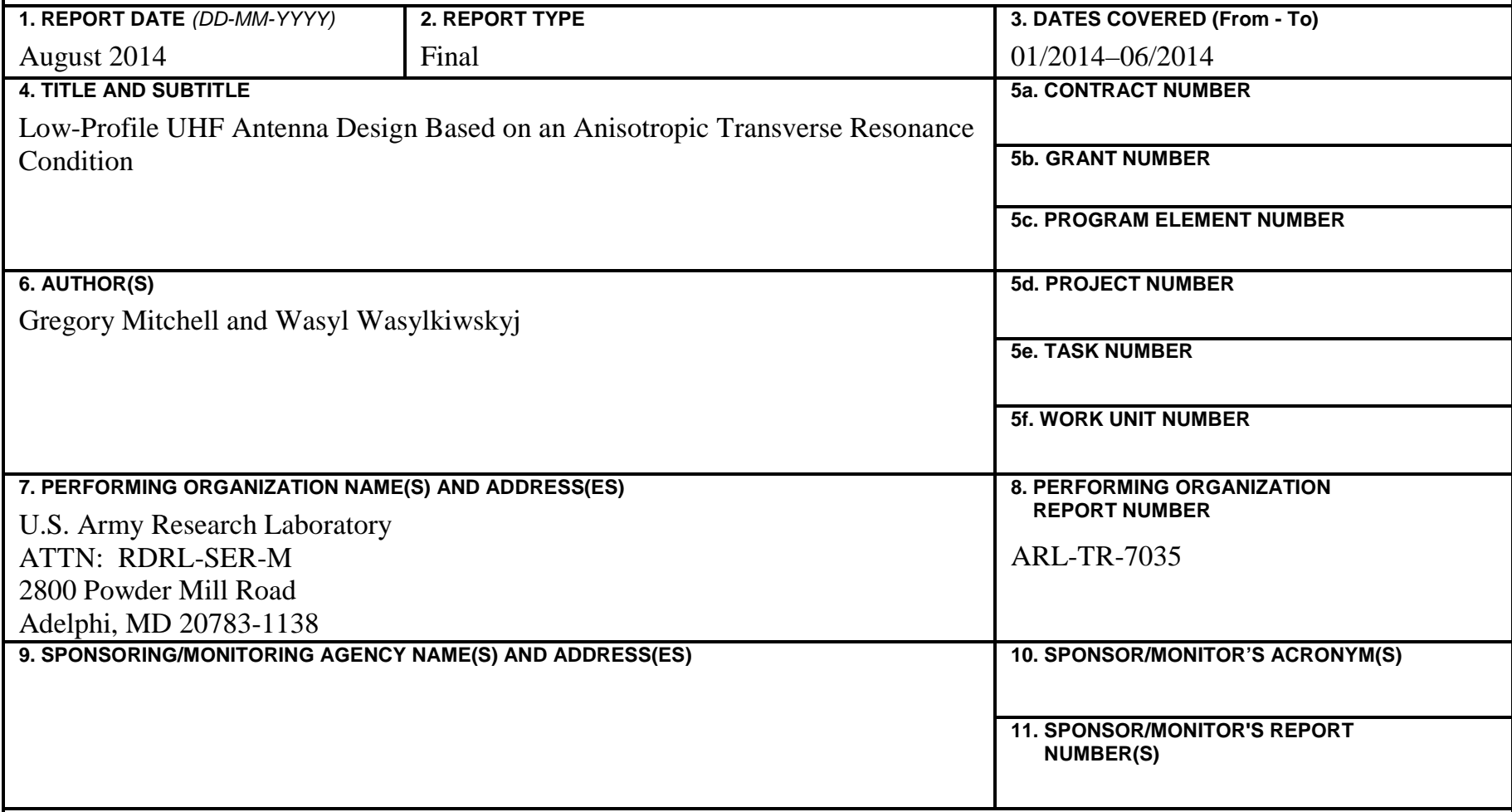

\section{DISTRIBUTION/AVAILABILITY STATEMENT}

Approved for public release; distribution unlimited.

\section{SUPPLEMENTARY NOTES}

\section{ABSTRACT}

A resonance condition is derived for a tapered rectangular cavity partially loaded with anisotropic magnetic material. This resonance condition leads to the design of an extremely low-profile $\left(\lambda_{0} / 18\right)$ antenna with over an octave of bandwidth and positive realized gain from 200-515 MHz. The equation derived for the shape of the partially loaded antenna cavity maintains a constant cutoff frequency even as more anisotropic material is introduced to the cavity. Also a method is determined to tailor the shape of the cavity as needed using the normal component of the permeability tensor with little to no effect on the performance of the antenna.

\section{SUBJECT TERMS}

anisotropic transverse resonance, low-profile antenna, metamaterials

\section{SECURITY CLASSIFICATION OF:}

A. Report

Unclassified

\section{b. ABSTRACT}

Unclassified c. THIS PAGE

Unclassified

\begin{tabular}{l|c} 
17. LIMITATION & 18. NUMBER \\
OF & OF \\
ABSTRACT & PAGES \\
UU & 32
\end{tabular}

19a. NAME OF RESPONSIBLE PERSON

Gregory Mitchell

19b. TELEPHONE NUMBER (Include area code) (301) 394-2322 


\section{Contents}

List of Figures $\quad$ iv

List of Tables $\quad$ v

$\begin{array}{ll}\text { 1. Introduction } & 1\end{array}$

2. Derivation of a Tapered Cavity Based on an Anisotropic Transverse Resonance 2

2.1 Anisotropic Transverse Resonance Derivation .........................................................

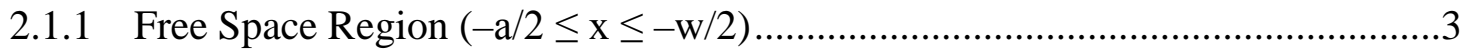

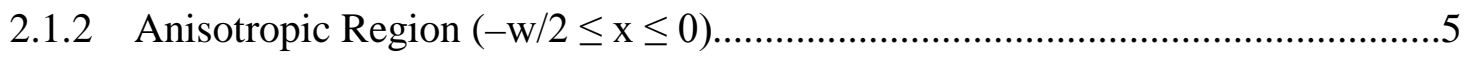

2.1.3 Solving the Boundary Conditions for the Impedances in the two Regions.........6

2.1.4 Solving for the Transverse Resonance Condition ............................................8

3. Low-Profile Antenna Design $\quad 10$

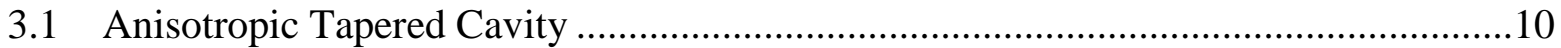

3.2 Anisotropic Non-Tapered Cavity .......................................................................14

3.3 Cascaded Antenna with Feed Structure......................................................................16

$\begin{array}{lr}\text { 4. Conclusions } & 19\end{array}$

$\begin{array}{lr}\text { 5. } & \text { References } \\ \end{array}$

$\begin{array}{ll}\text { Appendix. Werlatone } \mathbf{1 8 0}^{\circ} \text { Divider/Combiner } & 21\end{array}$

$\begin{array}{ll}\text { Distribution List } & 24\end{array}$ 


\section{List of Figures}

Fig. 1 The cavity geometry derived from the isotropic transverse resonance condition ${ }^{3} \ldots \ldots \ldots \ldots . .2$

Fig. 2 Transmission line representation of parallel plates partially loaded with anisotropic

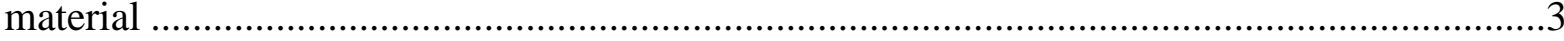

Fig. 3 Plot of Eq. 26 for various ratios of $\mu_{z} / \varepsilon_{y}$ with an assumed depth of 3.3 inches ...............10

Fig. 4 The cavity geometry derived from the anisotropic transverse resonance condition with $\mu_{z} / \varepsilon_{y}=15$

Fig. 5 Return loss at the probe inputs of the single-probe antenna (blue) and the symmetricprobe antenna (red)

Fig. 6 VSWR at the probe inputs of the single-probe antenna (blue) and the symmetricprobe antenna (red)

Fig. 7 Far field realized gain of the single-probe antenna (blue) and the symmetric-probe antenna (red)

Fig. 8 The cavity geometry derived from the anisotropic transverse resonance condition with $\mu_{z}=1$ and $\varepsilon_{y}=1$

Fig. $9 \mathrm{~S} 11$ at the probe inputs for the tapered antenna cavity with $\mu_{z}=15$ (blue) and the non-tapered antenna cavity with $\mu_{z}=1$ (red)

Fig. 10 VSWR at the probe inputs for the tapered antenna cavity with $\mu_{\mathrm{z}}=15$ (blue) and the non-tapered antenna cavity with $\mu_{z}=1$ (red)

Fig. 11 Realized gain for the tapered antenna cavity with $\mu_{z}=15$ (blue) and the non-tapered antenna cavity with $\mu_{z}=1$ (red)

Fig. 12 The diagram showing the connectivity between the $180^{\circ}$ coupler and the two-port antenna

Fig. 13 Return loss vs. frequency at for the antenna of Fig. 12 (red) and Fig. 8 (green)............17

Fig. 14 VSWR vs. frequency plots for the antenna of Fig 12 (red) and Fig. 4 (green)..............18

Fig. 15 Realized gain vs. frequency plots for the antenna of Fig 12 (red) and Fig. 4 (green) .....18

Fig. A-1 Picture of the $180^{\circ}$ coupler with the ports labeled. Port 1 is the input and ports 2 and 3 are the outputs.

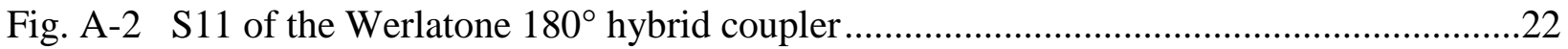

Fig. A-3 S21 and S31 of the Werlatone $180^{\circ}$ hybrid coupler ...............................................22

Fig. A-4 Phase difference at ports 2 and 3 of the $180^{\circ}$ coupler 


\section{List of Tables}

Table 1 The dimensions in inches for the geometry in Fig. 1..............................................2

Table 2 The dimensions in inches for the geometry in Fig. 4 ..............................................11 
INTENTIONALLY LEFT BLANK. 


\section{Introduction}

Resonating cavities have been used as a means to achieve stable and unidirectional radiation patterns for mounted antenna apertures. Radiation properties such as resonant frequency $\left(\boldsymbol{f}_{\boldsymbol{r}}\right)$ and realized gain are directly determined by the rectangular cavity dimensions. Generally, the $\boldsymbol{f}_{\boldsymbol{r}}$ is defined by the half-free space wavelength at the desired frequency, while the best gain results occur for a quarter-wavelength rectangular cavity in the normal direction.

Many applications in wireless communications and radar require antennas that conform to the surface of the supporting structure. Whenever applicable, the antenna aperture is approximated by a flat plane with the requirement that the dimension normal to the aperture ("profile") be minimized. Low-profile antennas (LPAs) are of special importance within the ultra-high frequencies (UHF) band, where they are used as communications antennas on military platforms. LPAs reduce platform visibility and decrease antenna weight, which becomes critically important in airborne platforms. In addition, many military vehicles contain several protruding antennas for multiple communication links at UHF. Therefore, there is great interest in developing LPAs with wide bandwidths (BWs). Meeting both requirements at the long wavelengths involved poses special difficulties.

Traditionally, magnetic materials have high losses at microwave frequencies above $100 \mathrm{MHz}$, which makes them unsuitable as substrates for UHF antenna structures. The availability of artificial magnetic metamaterials has widened the range of possible design approaches. Of particular interest are high index magnetic materials $\left(\mu_{r} \geq 10\right)$ that exhibit low loss in the UHF band. ${ }^{1,2}$ Furthermore, the development of artificial magnetic metamaterials allows engineers to separately control the values of both magnetic permeability and electric permittivity.

The goal of this report is to derive the anisotropic transverse resonance condition for a parallel plate structure partially loaded with anisotropic magneto-dielectric material. This calculation allows engineers to design cavity-backed LPAs that maintain a constant $\boldsymbol{f}_{r}$ when loaded with high index anisotropic materials. This is important because the refractive index of any material will alter $\boldsymbol{f}_{\boldsymbol{r}}$ when placed in a resonant cavity. By maintaining a constant $\boldsymbol{f}_{\boldsymbol{r}}$, the designer will suppress destructive interference from higher-order modes inside the cavity.

This report presents an antenna model where the profile reduction is achieved with high index anisotropic magnetic material. The antenna under investigation is a radiating, tapered rectangular cavity. An appropriately shaped high index magneto-dielectric material within the cavity establishes local resonance conditions with the conducting walls of the cavity. Furthermore, this report demonstrates that using anisotropic magnetic metamaterials to load this cavity achieves positive realized gain from $220-505 \mathrm{MHz}$ with a return loss (S11) of better than $-10 \mathrm{~dB}$ with a 
cavity profile depth of $0.06 \lambda_{r}$, where $\lambda_{r}$ is the cutoff free-space wavelength based on the dimensions of the aperture. All simulations are performed with CST Studio Suite 2014 and all materials are assumed to be lossless.

\section{Derivation of a Tapered Cavity Based on an Anisotropic Transverse Resonance}

A novel idea for a tapered rectangular cavity based on the transverse resonance was proposed in a previous report entitled "Comparison of Anisotropic versus Isotropic Metamaterials in a Low Profile UHF Antenna Design". ${ }^{3}$ This report presented an antenna based on a transverse resonance condition derived for isotropic materials and demonstrated the ability of this design to suppress higher-order modes.

The previous report showed that loading the cavity model shown in Fig. 1 with anisotropic material gave the best results, even though the transverse resonance condition that yielded this cavity was based on isotropic values of permeability and permittivity. The cavity dimensions that correspond to this design are given in Table 1.

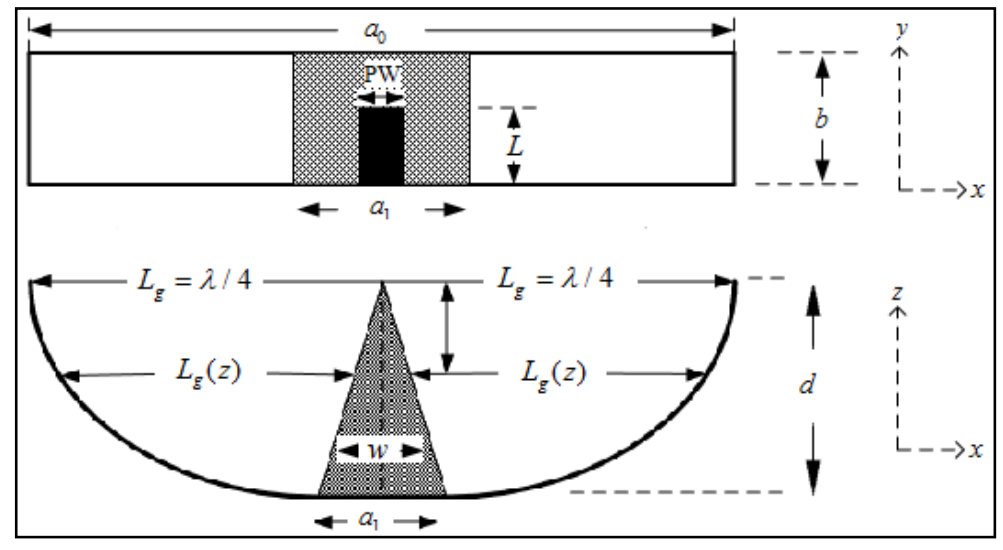

Fig. 1 The cavity geometry derived from the isotropic transverse resonance condition ${ }^{3}$

Table 1 The dimensions in inches for the geometry in Fig. 1

\begin{tabular}{|c|c|c|c|c|c|c|}
\hline $\boldsymbol{a}_{\boldsymbol{0}}$ & $\boldsymbol{b}$ & $\boldsymbol{a}_{\boldsymbol{1}}$ & $\boldsymbol{f}_{\boldsymbol{r}} \mathbf{( \mathbf { M H z } )}$ & $\boldsymbol{d}$ & $\boldsymbol{P W}$ & $\boldsymbol{L}$ \\
\hline 26.25 & 11.7 & 8.3 & 225 & 3.3 & 8.0 & 8.5 \\
\hline
\end{tabular}

Note that $a_{o}=\lambda_{r} / 2, b=a_{o} / 2.25$, and $a_{1}=a_{o} /\left[\left(\mu_{r} / \varepsilon_{r}\right)^{\wedge} 0.5\right]$. This design yielded a $\mathrm{S} 11<-6 \mathrm{~dB}$, or a voltage-standing-wave-ratio (VSWR) of better than 3:1, from 350-580 MHz, and a S11 $<-10 \mathrm{~dB}$, or a VSWR of better than 2:1, from 400-575 MHz. Furthermore, a positive 
realized gain is achieved from 220-580 MHz. These performance metrics are achieved with permeability and permittivity tensors of

$$
\mu_{r}=\left[\begin{array}{ccc}
15 & 0 & 0 \\
0 & 1 & 0 \\
0 & 0 & 1
\end{array}\right] \text { and } \varepsilon_{r}=\left[\begin{array}{ccc}
1 & 0 & 0 \\
0 & 1 & 0 \\
0 & 0 & 1
\end{array}\right] \text {. }
$$

Section 2.1 shows the results of the derivation of the anisotropic resonance condition for the anisotropic case, and its application to the improvement of the previous antenna design from Reference 3.

\subsection{Anisotropic Transverse Resonance Derivation}

At any point $z$ in the tapered cavity shown in Fig. 1, a resonance condition is established by material width $w(z)$ and the distance between the two conducting walls $a(z)$. At each point in space, this resembles a finite parallel plate waveguide partially loaded with anisotropic material with two conducting walls.

Figure 2 shows a transmission line representation of this parallel plate structure. For a known value of $w(z)$, we can determine the value of $a(z)$ in terms of $L_{g}$. The following subsections describe how to determine $L_{g}$ from the transverse resonance condition established by the conducting walls and the boundaries at the interface between the anisotropic material and free space.

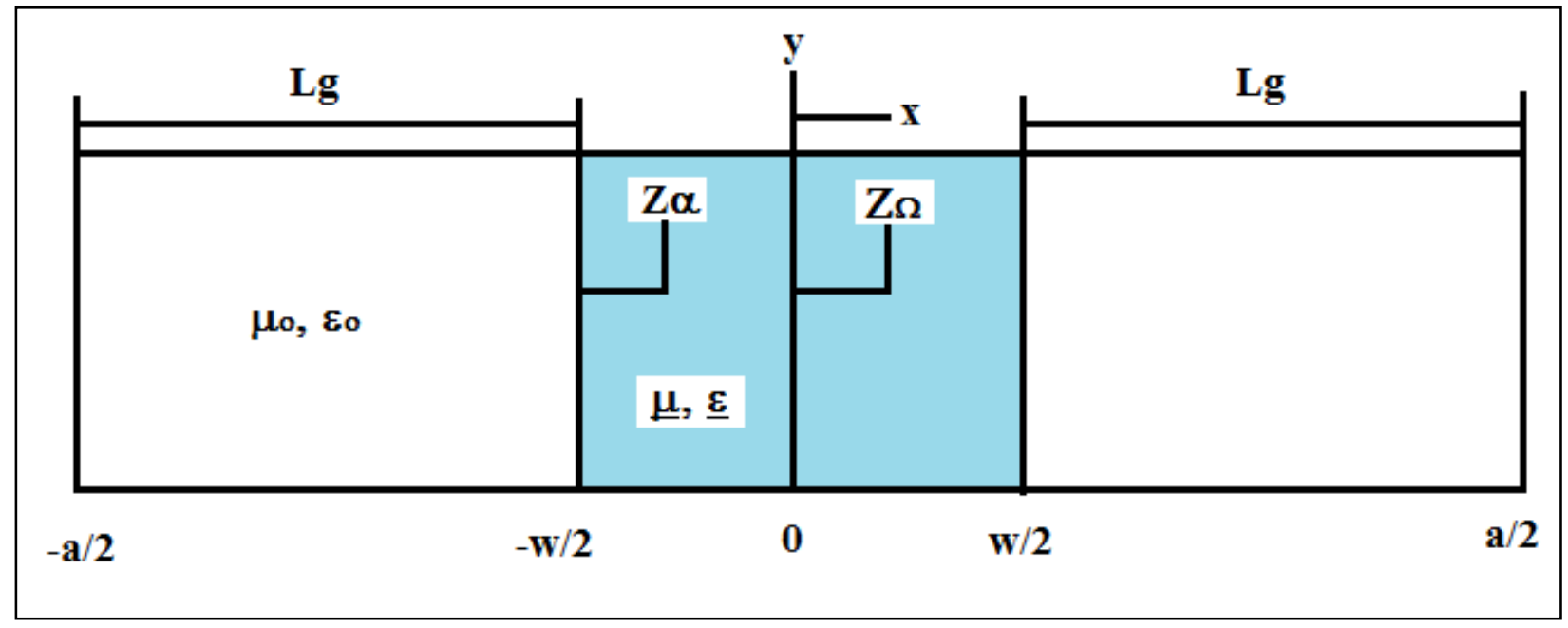

Fig. 2 Transmission line representation of parallel plates partially loaded with anisotropic material

\subsubsection{Free Space Region $(-a / 2 \leq x \leq-w / 2)$}

Assume Maxwell's source free equations: 


$$
\begin{gathered}
\nabla \times \underline{E}=-j \omega \mu_{o} \underline{H}, \\
\nabla \times \underline{H}=j \omega \varepsilon_{o} \underline{E} .
\end{gathered}
$$

Evaluating the curl operator of Eqs. 1a and 1b yields the following transverse components for the electric and magnetic fields in the waveguide in terms of $H_{z}$ and $E_{z}$ :

$$
\begin{gathered}
E_{x}=-\frac{j}{k_{o}^{2}-k_{z o}^{2}}\left(\omega \mu_{o} \frac{d H_{z}}{d y}+k_{z o} \frac{d E_{z}}{d x}\right), \\
E_{y}=\frac{j}{k_{o}^{2}-k_{z o}^{2}}\left(\omega \mu_{o} \frac{d H_{z}}{d x}-k_{z o} \frac{d E_{z}}{d y}\right), \\
H_{x}=\frac{j}{k_{o}^{2}-k_{z o}^{2}}\left(\omega \varepsilon_{o} \frac{d E_{z}}{d y}-k_{z o} \frac{d H_{z}}{d x}\right), \\
H_{y}=-\frac{j}{k_{o}^{2}-k_{z o}^{2}}\left(\omega \varepsilon_{o} \frac{d E_{z}}{d x}+k_{z o} \frac{d H_{z}}{d y}\right) .
\end{gathered}
$$

To solve for $H_{z}$, we formulate the transverse free space wave equation from Eqs. 1a and 1b as

$$
\begin{gathered}
\nabla_{T} \times \nabla_{T} \times \underline{H}=j \omega \varepsilon_{o}\left(\nabla_{T} \times \underline{E}\right)=\nabla_{T}\left(\nabla_{T} \cdot \underline{H}\right)-\nabla_{T}^{2} H_{z}, \\
j \omega \varepsilon_{o}\left(-j \omega \mu_{o} H_{z}\right)+\nabla_{T}^{2} H_{z}=0, \\
\left(\frac{d^{2} H_{z}}{d x^{2}}+\frac{d^{2} H_{z}}{d y^{2}}+k_{o}^{2}\right) H_{z}=0 .
\end{gathered}
$$

At the cutoff frequency $k_{z o}=0$, which allows us to calculate resonance at cutoff for this structure. If we assume a $\mathrm{TE}_{10}$-like resonance, then $k_{y o}=0$ for the first resonance at cutoff, which means no variation of the fields in the $y$-direction. This means that $\mathrm{d}^{2} H_{z} / \mathrm{dy}^{2}=0$ and

$$
\begin{gathered}
\frac{d^{2} H_{z}}{d x^{2}}+k_{o}^{2}=0, \\
\beta_{o}=k_{o} .
\end{gathered}
$$

Solving Eq. 4 for $H_{z}$ and inserting into Eq. 2b yields

$$
H_{z}=A e^{-j k_{0} x}+B e^{+j k_{0} x},
$$




$$
E_{y}=\frac{j \omega \mu_{o}}{k_{o}^{2}}\left(-j k_{o}\right)\left(A e^{-j k_{o} x}-B e^{+j k_{o} x}\right)=Z_{o}\left(A e^{-j k_{o} x}-B e^{+j k_{o} x}\right) .
$$

We can see from Eqs. 2a-2d that based on our resonance conditions on $E_{z}, k_{z o}$ and $k_{y o}$ that $E_{x}=0$, $H_{x}=0$, and $H_{y}=0$.

\subsubsection{Anisotropic Region $(-w / 2 \leq x \leq 0)$}

Again assume Maxwell's source free equations in the anisotropic region

$$
\begin{gathered}
\nabla \times \underline{E}=-j \omega \mu_{o} \underline{\underline{\mu_{r}}} \cdot \underline{H}, \\
\nabla \times \underline{H}=j \omega \varepsilon_{o} \underline{\underline{\varepsilon_{r}}} \cdot \underline{E},
\end{gathered}
$$

where the permeability and permittivity are now defined by tensors

$$
\underline{\underline{\mu_{r}}}=\left[\begin{array}{ccc}
\mu_{x} & 0 & 0 \\
0 & \mu_{y} & 0 \\
0 & 0 & \mu_{z}
\end{array}\right] \text { and } \underline{\underline{\varepsilon_{r}}}=\left[\begin{array}{ccc}
\varepsilon_{x} & 0 & 0 \\
0 & \varepsilon_{y} & 0 \\
0 & 0 & \varepsilon_{z}
\end{array}\right] \text {. }
$$

Evaluating the curl operator of Eqs. 7a and 7b yields the following transverse components for the electric and magnetic fields in the waveguide in terms of $H_{z}$ and $E_{z}^{4,5}$

$$
\begin{aligned}
& E_{x}=-\frac{j}{k_{o}^{2} \mu_{y} \varepsilon_{x}-k_{z 1}^{2}}\left(\omega \mu_{o} \mu_{y} \frac{d H_{z}}{d y}+k_{z 1} \frac{d E_{z}}{d x}\right), \\
& E_{y}=\frac{j}{k_{o}^{2} \mu_{x} \varepsilon_{y}-k_{z 1}^{2}}\left(\omega \mu_{o} \mu_{x} \frac{d H_{z}}{d x}-k_{z 1} \frac{d E_{z}}{d y}\right), \\
& H_{x}=\frac{j}{k_{o}^{2} \mu_{x} \varepsilon_{y}-k_{z 1}^{2}}\left(\omega \varepsilon_{o} \varepsilon_{y} \frac{d E_{z}}{d y}-k_{z 1} \frac{d H_{z}}{d x}\right), \\
& H_{y}=-\frac{j}{k_{o}^{2} \mu_{y} \varepsilon_{x}-k_{z 1}^{2}}\left(\omega \varepsilon_{o} \varepsilon_{x} \frac{d E_{z}}{d x}+k_{z 1} \frac{d H_{z}}{d y}\right) .
\end{aligned}
$$

To solve for $H_{z}$ we formulate the transverse anisotropic wave equation from Eqs. 7a and 7b: ${ }^{4,5}$

$$
\begin{gathered}
\nabla_{T} \times \nabla_{T} \times \underline{H}=j \omega \underline{\underline{\varepsilon_{o}}} \underline{\underline{\varepsilon_{r}}} \cdot\left(\nabla_{T} \times \underline{E}\right), \\
\nabla_{T} \times \nabla_{T} \times \underline{H}=j \omega \underline{\underline{\varepsilon_{o}}} \underline{\underline{\varepsilon_{r}}} \cdot\left(-j \omega \mu_{o} \underline{\underline{\mu_{r}}} \cdot \underline{H}\right),
\end{gathered}
$$




$$
\begin{gathered}
\nabla_{T} \times{\underline{\varepsilon_{r}}}^{-1} \cdot\left(\nabla_{T} \times \underline{H}\right)=k_{o}^{2} \underline{\underline{\mu_{r}}} \cdot \underline{H}, \\
\frac{k_{o}^{2} \mu_{x}}{k_{o}^{2} \mu_{x} \varepsilon_{y}-k_{z 1}^{2}} \frac{d^{2} H_{z}}{d x^{2}}+\frac{k_{o}^{2} \mu_{y}}{k_{o}^{2} \mu_{y} \varepsilon_{x}-k_{z 1}^{2}} \frac{d^{2} H_{z}}{d y^{2}}+k_{o}^{2} \mu_{z} H_{z}=0 .
\end{gathered}
$$

Now set $k_{z 1}=0$, which allows us to calculate resonance at cutoff for this structure. Similarly, we can assume that $k_{y 1}=0$ for the first resonance at cutoff. This means that $\mathrm{d}^{2} H_{z} / \mathrm{dy}^{2}=0$

$$
\begin{gathered}
\frac{d^{2} H_{z}}{d x^{2}}+k_{o}^{2} \mu_{z} \varepsilon_{y}=0 \\
\beta_{1}=k_{o} \sqrt{\mu_{z} \varepsilon_{y}} .
\end{gathered}
$$

Solving Eq. 11 for $H_{z}$ yields

$$
\begin{gathered}
H_{z}=C e^{-j \beta_{1} x}+D e^{+j \beta_{1} x}, \\
E_{y}=\frac{j Z_{o}}{k_{o} \varepsilon_{y}}\left(-j \beta_{1}\right)\left(C e^{-j \beta_{1} x}-D e^{+j \beta_{1} x}\right)=Z_{o} \sqrt{\frac{\mu_{z}}{\varepsilon_{y}}}\left(C e^{-j \beta_{1} x}-D e^{+j \beta_{1} x}\right) .
\end{gathered}
$$

We can see from Eqs. 9a-9d that based on our resonance conditions on $E_{z}, k_{z 1}$ and $k_{y 1}$ that $E_{x}=0$, $H_{x}=0$ and $H_{y}=0$.

\subsubsection{Solving the Boundary Conditions for the Impedances in the two Regions}

The first boundary condition exists at the perfect electric conductor (PEC) boundary at $x=-a / 2$ where the electric field is known to be zero:

$$
\begin{gathered}
\left.E_{y}\right|_{x=-\frac{a}{2}}=0 \rightarrow A e^{j k_{o} \frac{a}{2}}=B e^{-j k_{o} \frac{a}{2}}, \\
A=B e^{-j k_{o} a} .
\end{gathered}
$$

Plugging Eq. 14 into our Eqs. 6a and 6b yields

$$
\begin{aligned}
E_{y} & =Z_{o} B\left[e^{-j k_{o} x} e^{-j k_{o} a}-e^{+j k_{o} x}\right] \\
& =Z_{o} B e^{-j k_{o} \frac{a}{2}}\left[e^{-j k_{o}\left(x+\frac{a}{2}\right)}-e^{+j k_{o}\left(x+\frac{a}{2}\right)}\right], \\
E_{y} & =-2 j Z_{o} B e^{-j k_{o} \frac{a}{2}} \sin \left[k_{o}\left(x+\frac{a}{2}\right)\right] .
\end{aligned}
$$


Similarly,

$$
\begin{gathered}
H_{z}=B e^{-j k_{o} a} e^{-j k_{o} x}+B e^{+j k_{o} x}, \\
H_{z}=2 B e^{-j k_{o} \frac{a}{2}} \cos \left[k_{o}\left(x+\frac{a}{2}\right)\right] .
\end{gathered}
$$

Now we can solve for the impedance of the free space region as $Z=-E_{y} / H_{z}$

$$
Z_{o}=-\frac{E_{y}}{H_{z}}=j Z_{o} \tan \left[k_{o}\left(x+\frac{a}{2}\right)\right], 0 \leq\left(x+\frac{a}{2}\right) \leq \frac{a-w}{2} \text {. }
$$

The second boundary condition exists at $x=-w / 2$, where the tangential fields at the boundary are equal. In this case, there are two tangential fields in $E_{y}$ and $H_{z}$. At the boundary, we have the following three conditions:

$$
\begin{aligned}
& \left.E_{y}^{-}\right|_{x=-\frac{w}{2}}=\left.E_{y}^{+}\right|_{x=-\frac{w}{2}}, \\
& \left.H_{z}^{-}\right|_{x=-\frac{w}{2}}=\left.H_{z}^{+}\right|_{x=-\frac{w}{2}}, \\
& \left.Z_{o}\right|_{x=-\frac{w}{2}} ^{-}=\left.Z_{1}\right|_{x=-\frac{w}{2}} ^{+} .
\end{aligned}
$$

Plugging Eqs. 13 and 15 into Eqs. 17a and 17b yields the following set of equations:

$$
\begin{gathered}
-2 j B e^{-j k_{o} \frac{a}{2}} \sin \left[k_{o}\left(\frac{a-w}{2}\right)\right]=\sqrt{\frac{\mu_{z}}{\varepsilon_{y}}}\left(C e^{-j \beta_{1} \frac{w}{2}}-D e^{+j \beta_{1} \frac{w}{2}}\right), \\
2 B e^{-j k_{o} \frac{a}{2}} \cos \left[k_{o}\left(\frac{a-w}{2}\right)\right]=C e^{-j \beta_{1} \frac{w}{2}}-D e^{+j \beta_{1} \frac{w}{2}} .
\end{gathered}
$$

This gives us two equations to solve for three unknowns. In order to solve for the third unknown, we can match Eq. 16 to the impedance in the anisotropic region at $x=-w / 2$. Again we solve for $Z=-E_{y} / H_{z}$ from Eqs. 15a and 15b:

$$
\begin{gathered}
Z_{1}=Z_{o} \sqrt{\frac{\mu_{z}}{\varepsilon_{y}}}\left[\frac{D e^{+j \beta_{1} x}-C e^{-j \beta_{1} x}}{D e^{+j \beta_{1} x}+C e^{-j \beta_{1} x}}\right]=Z_{o} \sqrt{\frac{\mu_{z}}{\varepsilon_{y}}}\left[\frac{1-\rho e^{-j 2 \beta_{1} x}}{1+\rho e^{-j 2 \beta_{1} x}}\right],-\frac{w}{2} \leq x \leq 0, \\
\left|Z_{1}\right|=Z_{o} \sqrt{\frac{\mu_{z}}{\varepsilon_{y}}},
\end{gathered}
$$




$$
\rho=\frac{C}{D} \text {. }
$$

Now applying boundary condition Eq. 17c to Eqs. 16 and 20

$$
\begin{gathered}
Z_{o} \sqrt{\frac{\mu_{z}}{\varepsilon_{y}}}\left[\frac{1-\rho e^{j \beta_{1} w}}{1+\rho e^{j \beta_{1} w}}\right]=j Z_{o} \tan \left[k_{o}\left(\frac{a-w}{2}\right)\right], \\
{\left[\frac{1-\rho e^{j \beta_{1} w}}{1+\rho e^{j \beta_{1} w}}\right]=j \sqrt{\frac{\varepsilon_{y}}{\mu_{z}}} \tan \left[k_{o}\left(\frac{a-w}{2}\right)\right]=j \bar{X},} \\
1-\rho e^{j \beta_{1} w}=j \bar{X}+j \bar{X} \rho e^{j \beta_{1} w}, \\
1-j \bar{X}=j \bar{X} \rho e^{j \beta_{1} w}+\rho e^{j \beta_{1} w}, \\
\rho=\frac{1-j \bar{X}}{1+j \bar{X}} e^{-j \beta_{1} w}=e^{-j 2 \pi \sqrt{\mu_{z} \varepsilon_{y}} \frac{w}{\lambda}}\left\{\frac{1-j \sqrt{\frac{\varepsilon_{y}}{\mu_{z}}} \tan \left[\pi\left(\frac{a-w}{\lambda}\right)\right]}{1+j \sqrt{\frac{\varepsilon_{y}}{\mu_{z}}} \tan \left[\pi\left(\frac{a-w}{\lambda}\right)\right]}\right\} .
\end{gathered}
$$

Substituting Eq. 22 into Eq. 21 gives us our third equation along with Eqs. 18 and 19 to solve for the three unknowns $B, C$, and $D$.

\subsubsection{Solving for the Transverse Resonance Condition}

If we view Fig. 2 as a transmission line representation of our problem, we can solve for $L_{g}$ in terms of $w$ for a given wavelength $\left(\lambda_{o}\right)$. For instance, at $200 \mathrm{MHz}, \lambda_{o}=1.5 \mathrm{~m}$. We can use the input impedance transformations of transmission line theory to calculate $\vec{Z}_{\text {in }}$ at $x=0$. Then by

symmetry the transverse resonance condition simplifies to $\vec{Z}_{\text {in }}=0$ or $\vec{Y}_{\text {in }}=0$.

Starting at $x=-a / 2$, we can calculate $\vec{Z}_{\text {in }}$ at $x=-w / 2$ by

$$
\vec{Z}_{\alpha}=j Z_{o} \tan \left[k_{o} L_{g}\right] \text {. }
$$

We can now calculate $\vec{Z}_{\text {in }}$ at $x=0$ as

$$
\vec{Z}_{\Omega}=Z_{1} \frac{\vec{Z}_{\alpha}+j Z_{1} \tan \left(\beta_{1} \frac{w}{2}\right)}{Z_{1}+j \vec{Z}_{\alpha} \tan \left(\beta_{1} \frac{w}{2}\right)}
$$

The transverse resonance condition simplifies Eq. 24 to 


$$
Z_{1}+j \vec{Z}_{\alpha} \tan \left(\beta_{1} \frac{w}{2}\right)=0
$$

Plugging Eqs. 20b and 23 into Eq. 25 yields the following equation for $L_{g}$ :

$$
\begin{gathered}
Z_{o} \sqrt{\frac{\mu_{z}}{\varepsilon_{y}}}-Z_{o} \tan \left(k_{o} L_{g}\right) \tan \left(k_{o} \sqrt{\mu_{z} \varepsilon_{y}} \frac{w}{2}\right)=0 \\
\frac{L_{g}}{\lambda}=\frac{1}{2 \pi} \tan ^{-1}\left[\frac{\sqrt{\frac{\mu_{z}}{\varepsilon_{y}}}}{\tan \left(\frac{\pi w}{\lambda} \sqrt{\mu_{z} \varepsilon_{y}}\right)}\right] .
\end{gathered}
$$

In Reference 3, $L_{g}$ depended on both $\mu_{r}$ and $\varepsilon_{r}$, which means all six permittivity and permeability tensor elements affected the return loss of the antenna. We know that ultimately the permeability value in the direction of the magnetic field at the aperture is what determines the best return loss for our antenna in Fig. 1. ${ }^{3}$ Now we see that as we change this tensor value $\left(\mu_{x}\right)$, there will be no effect on the taper of the cavity.

Figure 3 shows the relationship between the ratio of $\mu_{z} / \varepsilon_{y}$ and the shape of the cavity. Note that for a ratio of 1 , we have a purely linear taper. Now that we have an explicit expression for the cavity taper based on anisotropic permittivity and permeability, we apply it to antenna design in Fig. 1 to see if we can further optimize the performance. 


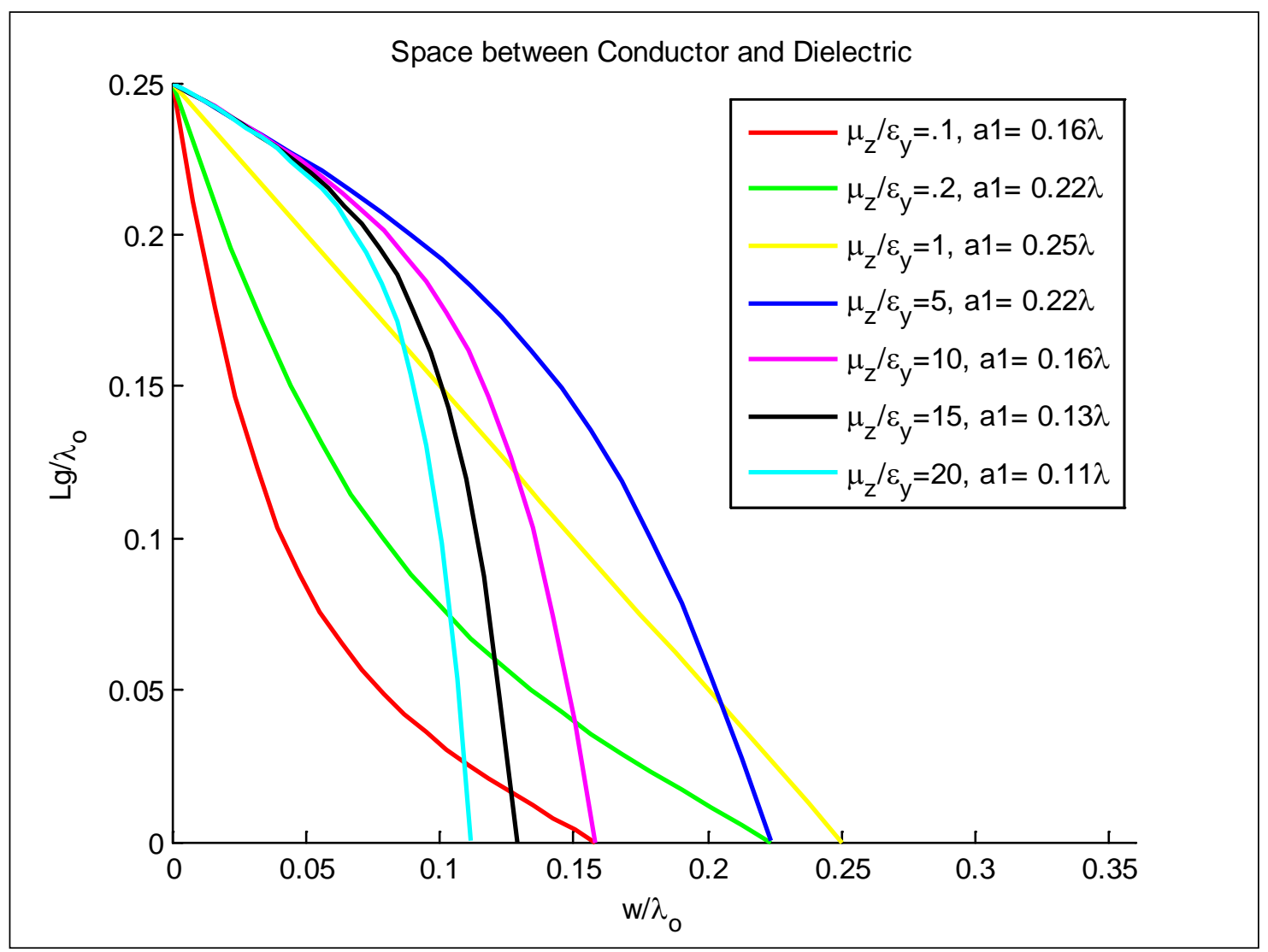

Fig. 3 Plot of Eq. 26 for various ratios of $\mu_{z} / \varepsilon_{y}$ with an assumed depth of 3.3 inches

\section{Low-Profile Antenna Design}

Now we can use Eq. 26 to design an optimum cavity taper for our LPA. We start out by comparing a tapered cavity based on our anisotropic transverse resonance condition to that of an antenna in Fig. 1.

\subsection{Anisotropic Tapered Cavity}

Figure 1 has a cavity shape based on a ratio of $\mu_{r} / \varepsilon_{r}=15 .^{3}$ Therefore, for an accurate comparison using the same cavity shape, we use a ratio of $\mu_{z} / \varepsilon_{y}=15$. Furthermore, we know that a value of $\mu_{x}=15$ gives our best broadband return loss. ${ }^{3}$ This results in the following permeability and permittivity tensors for our anisotropic material: 


$$
\mu_{r}=\left[\begin{array}{ccc}
15 & 0 & 0 \\
0 & 1 & 0 \\
0 & 0 & 15
\end{array}\right] \text { and } \varepsilon_{r}=\left[\begin{array}{lll}
1 & 0 & 0 \\
0 & 1 & 0 \\
0 & 0 & 1
\end{array}\right] .
$$

Figure 4 shows a cavity that is very similar to Fig. 1 . The dimensions of the tapered cavity are given in Table 2. Note that now $a_{1}=a_{o} /\left[\left(\mu_{z} / \varepsilon_{y}\right)^{\wedge} 0.5\right]$. In order to achieve the best wideband return loss, $\boldsymbol{f}_{\boldsymbol{r}}$ has been lowered to $150 \mathrm{MHz}$. This results in a much larger aperture; however, the profile of the antenna remains $d=3.3$ inches.

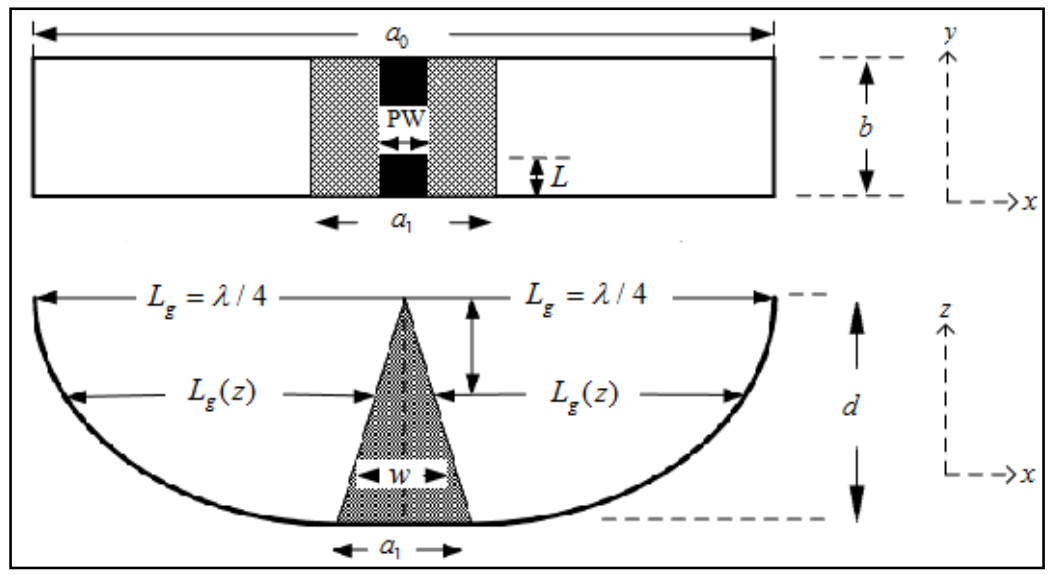

Fig. 4 The cavity geometry derived from the anisotropic transverse resonance condition with $\mu_{z} / \varepsilon_{y}=15$

Table 2 The dimensions in inches for the geometry in Fig. 4

\begin{tabular}{|c|c|c|c|c|c|c|}
\hline $\boldsymbol{a}_{\boldsymbol{0}}$ & $\boldsymbol{b}$ & $\boldsymbol{a}_{\boldsymbol{1}}$ & $\boldsymbol{f}_{\boldsymbol{r}}(\mathbf{M H z})$ & $\boldsymbol{d}$ & $\boldsymbol{P W}$ & $\boldsymbol{L}$ \\
\hline 39.4 & 17.5 & 10.2 & 150 & 3.3 & $0.25 \lambda_{r}$ & $0.35 \lambda_{r}$ \\
\hline
\end{tabular}

This antenna model is now a two-port system with two symmetric probes. This differs from the one-port probe shown in Fig. 1. Feeding the two probes $180^{\circ}$ out of phase provides a continuous current path across a positive-to-negative voltage potential across the walls of the cavity in the $y$-direction. This should provide a better impedance match at the input to both ports.

Figure 5 shows the return loss versus frequency for the single-port antenna of Fig. 1 and the symmetric-port antenna of Fig. 4. This shows a S11 $<-6 \mathrm{~dB}$ from $230-505 \mathrm{MHz}$, which is about an $80-\mathrm{MHz}$ improvement in $\mathrm{BW}$ at the upper end of the frequency range. We also have a much flatter S11 response from 250-420 MHz even though the return loss is better for the single-probe antenna. Figure 6 shows the VSWR versus frequency for the single-port antenna of Fig. 1 and the symmetric-port antenna of Fig. 4. This shows better than a 3:1 VSWR from 230-505 MHz, 
which is about an 80-MHz improvement in bandwidth at the upper end of the frequency range of the single probe antenna. Figure 7 shows the realized gain versus frequency for the single-port antenna of Fig. 1 and the symmetric-port antenna of Fig. 4. This shows the most dramatic difference in performance. Due to the flat return loss response of the symmetric-probe antenna over $420-500 \mathrm{MHz}$, the deep trough in the realized gain for the single-probe antenna is eliminated. This gives us a positive realized gain in excess of the entire BW 180-515 MHz.

The combination of realized gain and return loss yields over an octave of BW for the antenna design in Fig. 4 with tensor values of Eq. 27. However, we have forced the $\mu_{z}$ component of the permeability tensor to be 15 . However, the $\mu_{z}$ component should have little effect on the overall performance of the antenna. ${ }^{3}$ Section 3.2 investigates how setting $\mu_{z}=1$ affects the shape of the cavity and the performance of the antenna.

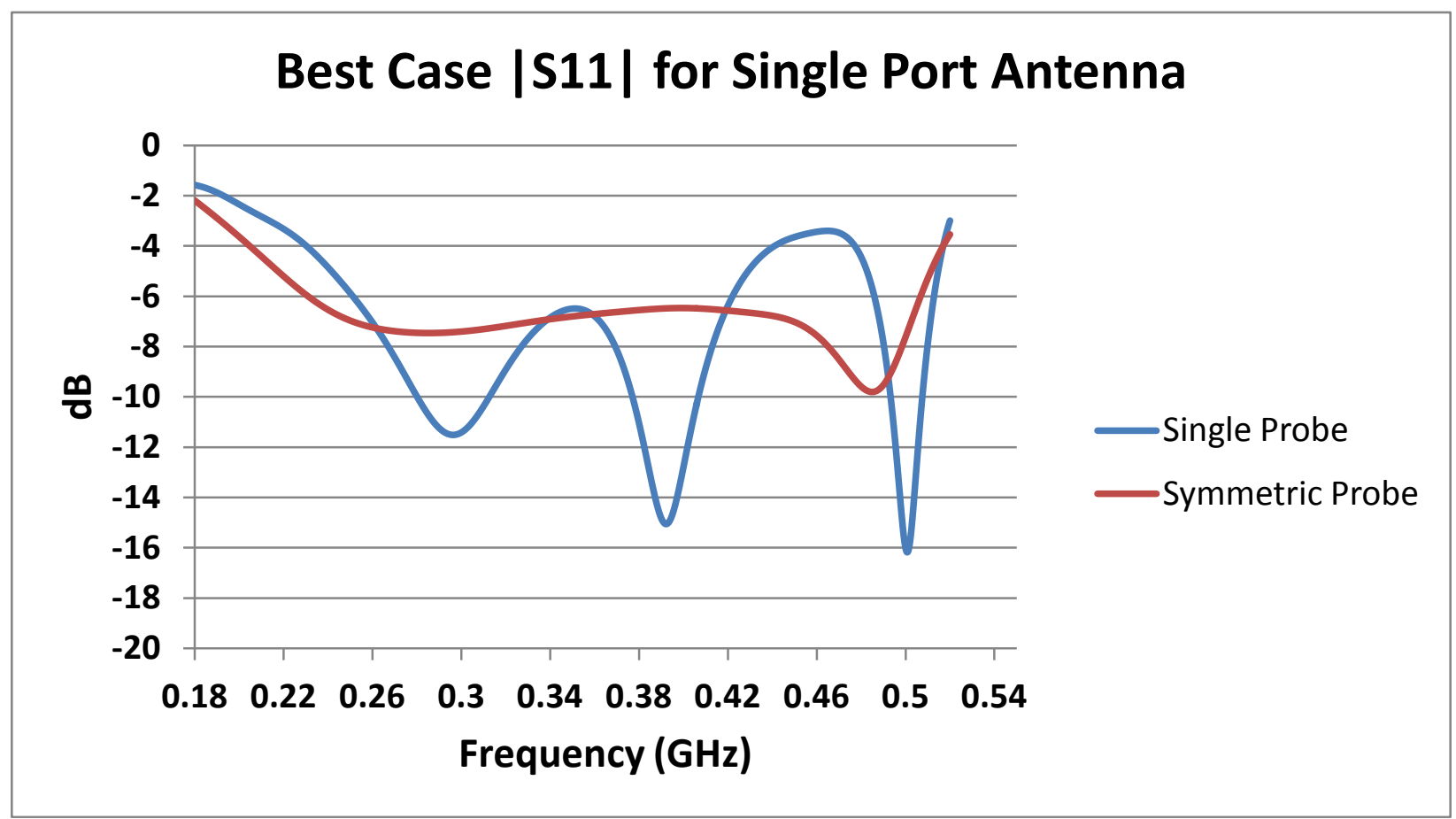

Fig. 5 Return loss at the probe inputs of the single-probe antenna (blue) and the symmetric-probe antenna (red) 


\section{Best Case VSWR for Single and Symmetric Probe Antenna}

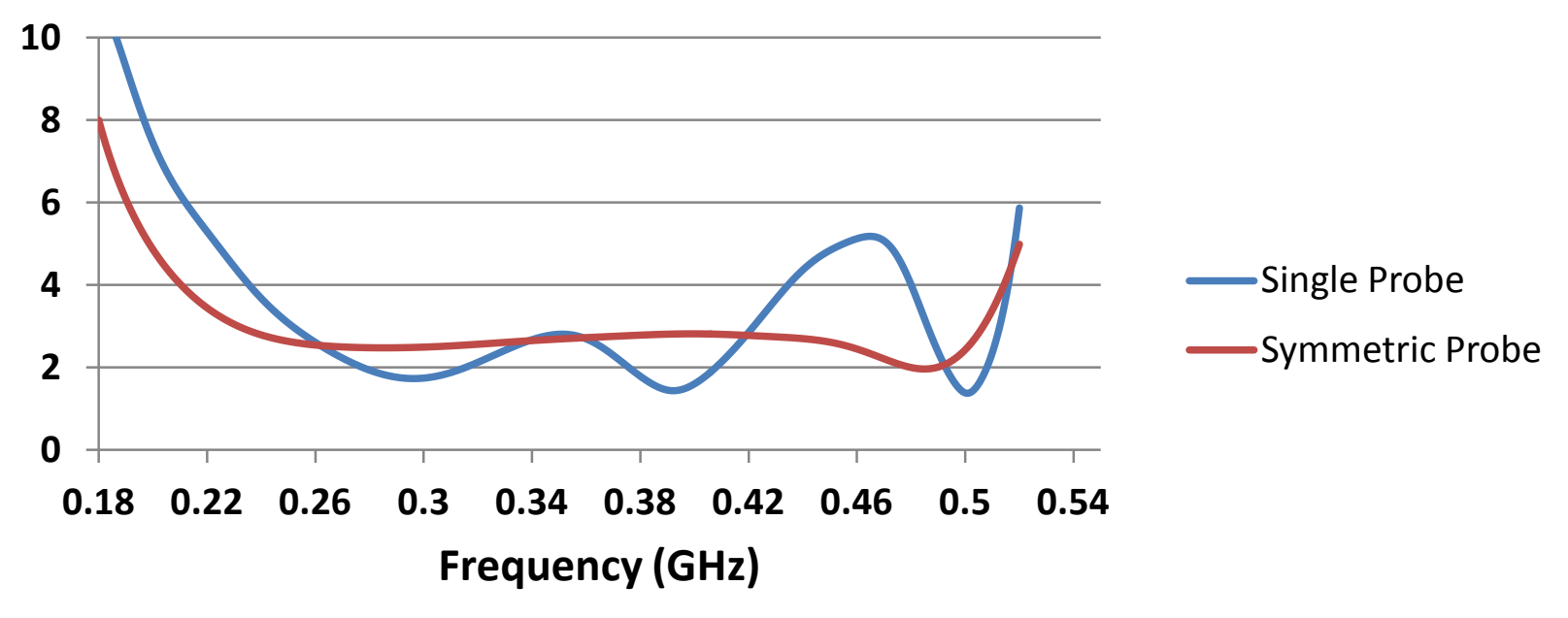

Fig. 6 VSWR at the probe inputs of the single-probe antenna (blue) and the symmetric-probe antenna (red)

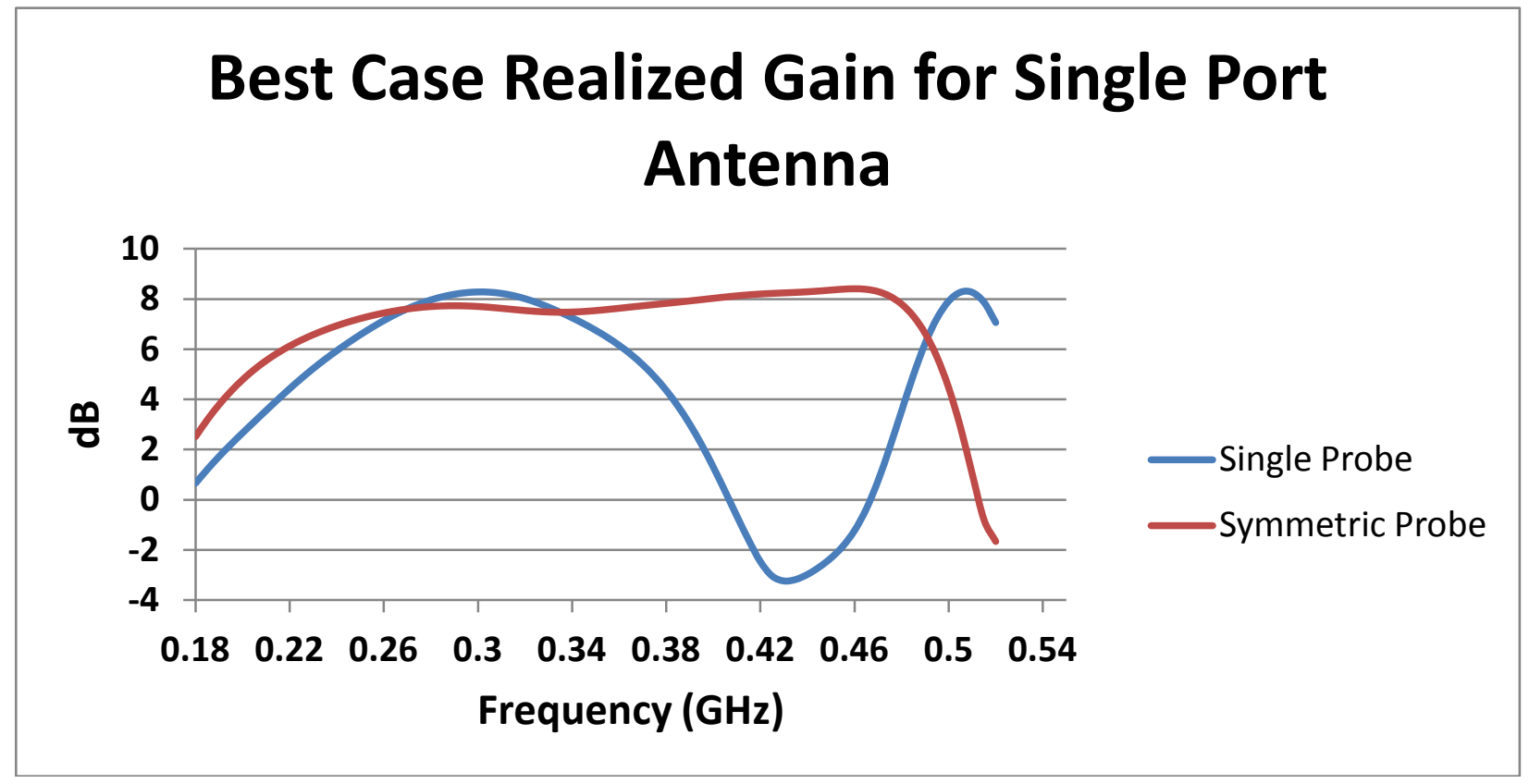

Fig. 7 Far field realized gain of the single-probe antenna (blue) and the symmetric-probe antenna (red) 


\subsection{Anisotropic Non-Tapered Cavity}

This section explores how setting $\mu_{\mathrm{z}}=1$ affects the cavity shape of the antenna design. The results of Reference 3 suggest that this should have little effect on the return loss of the antenna. Figure 3 suggests that a ratio of $\mu_{z} / \varepsilon_{y}=1$ will result in a linearly tapered cavity. However, for the special case of $\mu_{z} / \varepsilon_{y}=1$, where both $\mu_{z}$ and $\varepsilon_{y}$ are unity, the walls of the cavity do not need to be tapered at all.

Figure 8 shows the cavity geometry given by Eq. 26 for the following permeability and permittivity tensors

$$
\mu_{r}=\left[\begin{array}{ccc}
15 & 0 & 0 \\
0 & 1 & 0 \\
0 & 0 & 1
\end{array}\right] \text { and } \varepsilon_{r}=\left[\begin{array}{ccc}
1 & 0 & 0 \\
0 & 1 & 0 \\
0 & 0 & 1
\end{array}\right]
$$

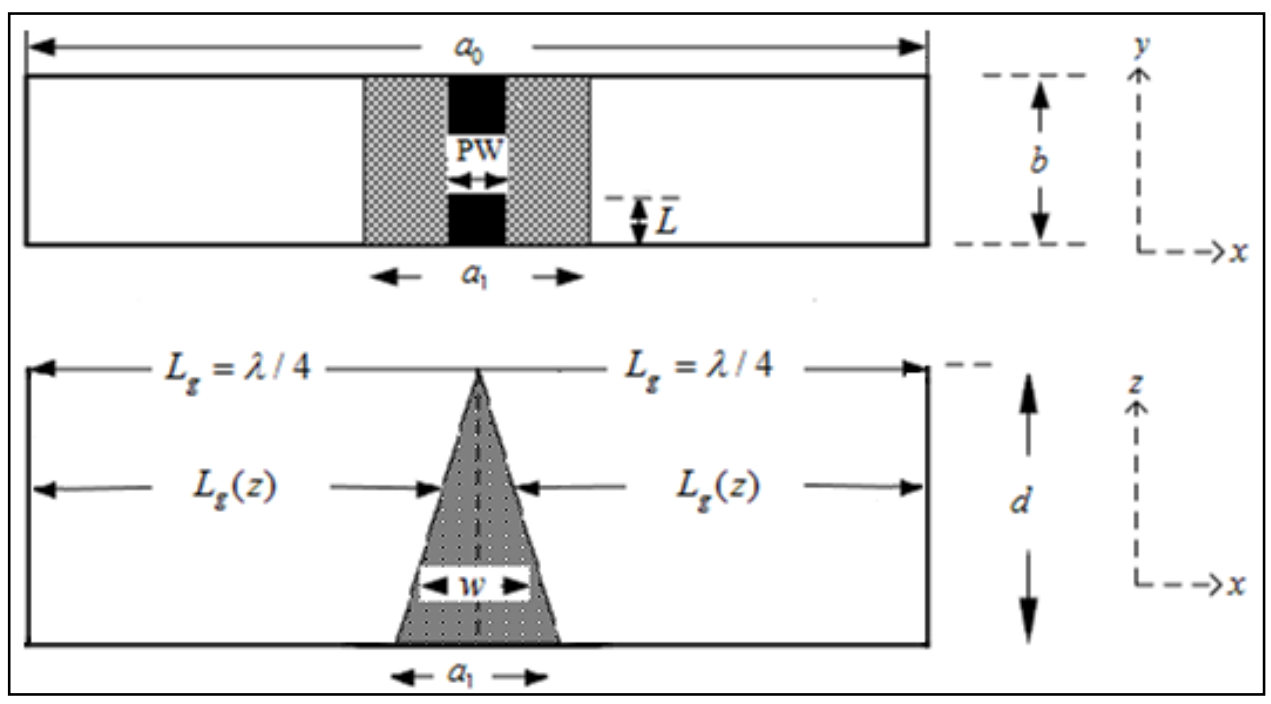

Fig. 8 The cavity geometry derived from the anisotropic transverse resonance condition with $\mu_{z}=1$ and $\varepsilon_{y}=1$

The cavity shape is a rectangular cavity with no taper. This is a very different result from those of Figs. 1 and 4. This indicates that regardless of the anisotropic tensor values, as long as $\mu_{\mathrm{z}}=1$ and $\varepsilon_{y}=1$, any amount of loading will result in the same cavity shape and a constant $f_{r}$ even at the material to free space boundary. We now see if changing the value of $\mu_{z}$ from 15 to 1 has any effect on the return loss or realized gain of the antenna.

Figures 9-11 show very good agreement in the return loss, VSWR, and realized gain plots. Therefore, we conclude that the non-tapered cavity shape of Fig. 8 has no noticeable effect on the overall performance of the antenna. This is a very useful result, because it means the shape of 
the cavity can be changed to fit in different environments as long as the designer has some amount of control over the $\mu_{z}$ component of the permeability tensor.

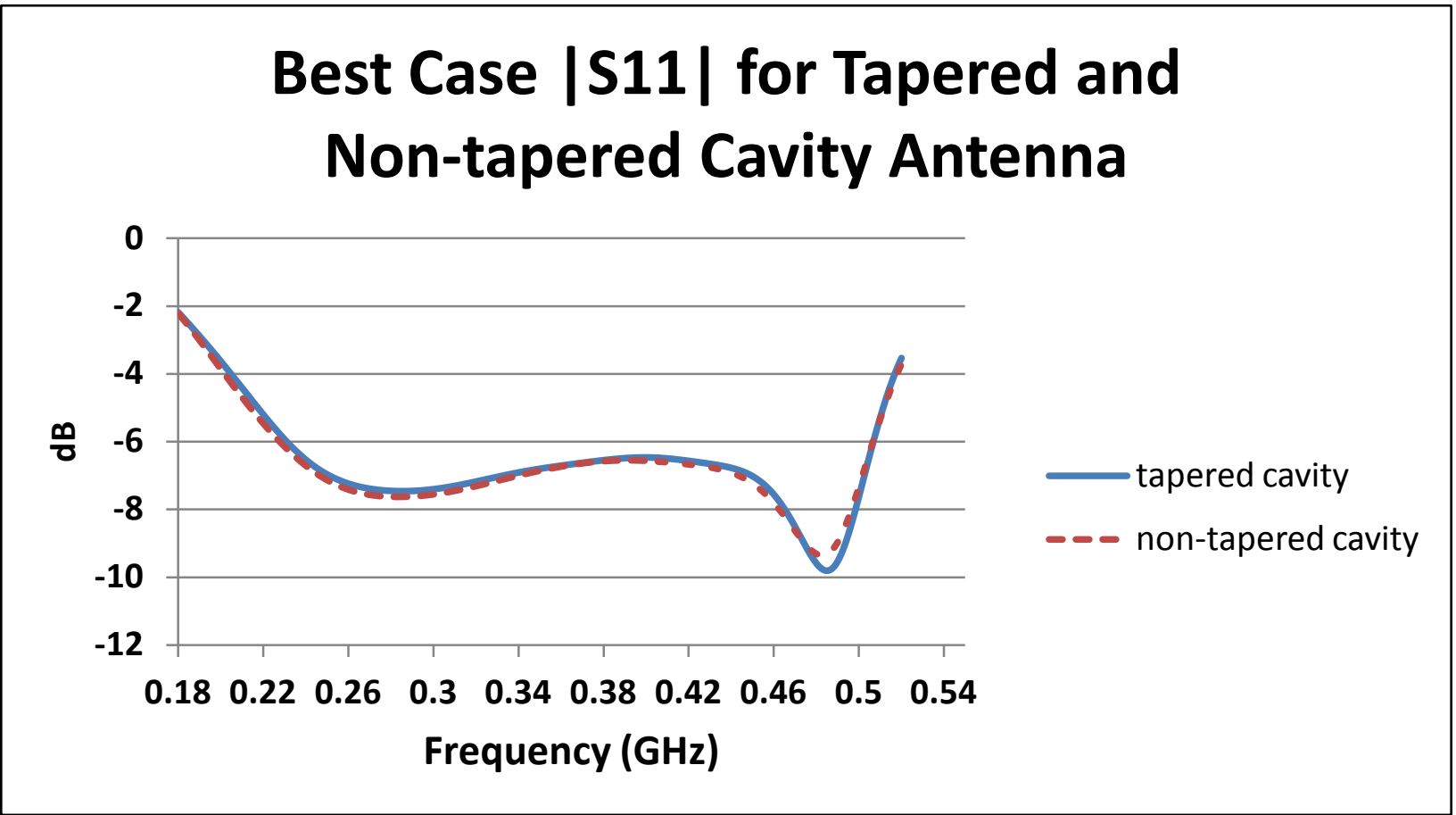

Fig. 9 S11 at the probe inputs for the tapered antenna cavity with $\mu_{z}=15$ (blue) and the non-tapered antenna cavity with $\mu_{\mathrm{z}}=1$ (red)

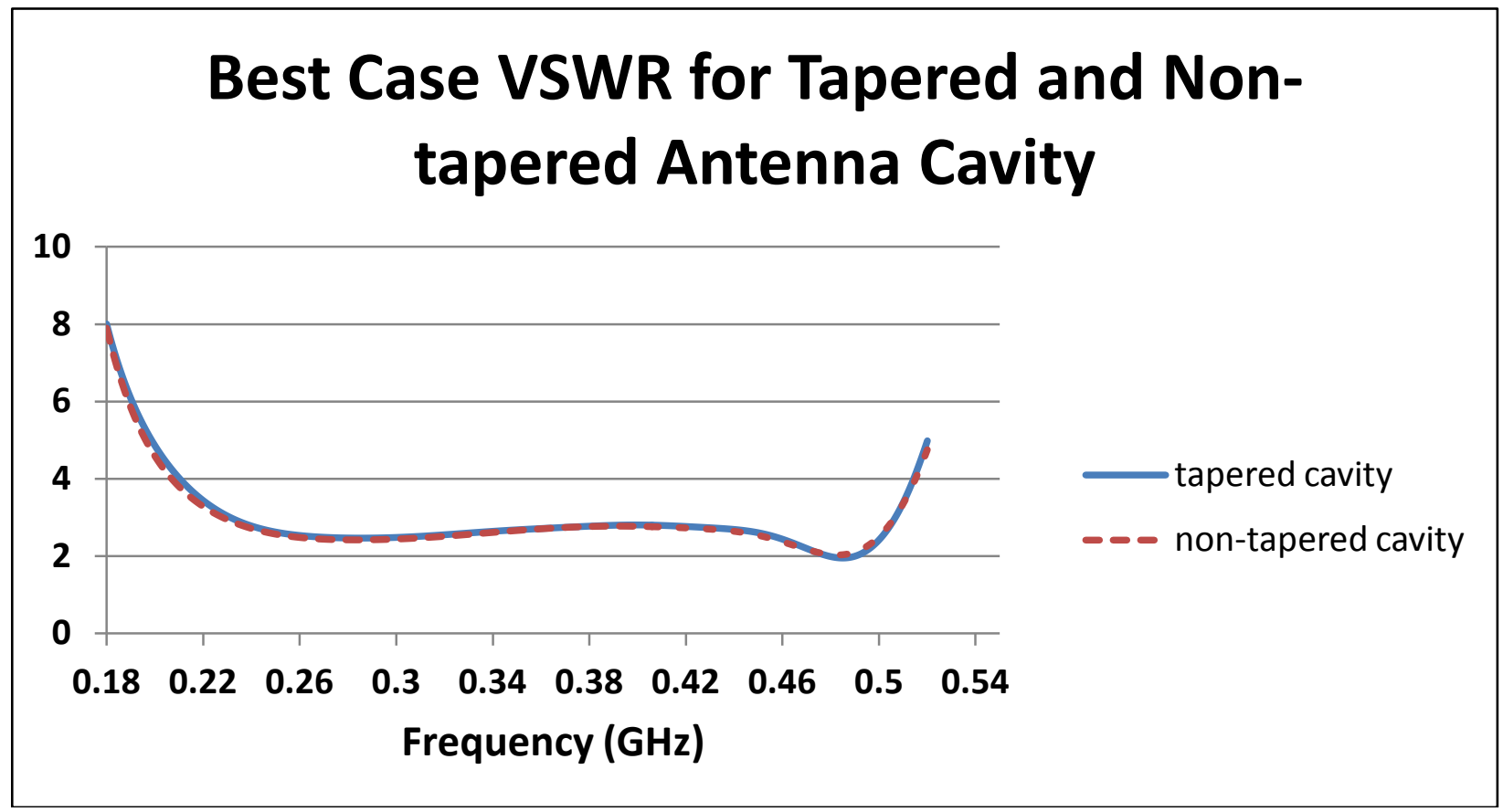

Fig. 10 VSWR at the probe inputs for the tapered antenna cavity with $\mu_{z}=15$ (blue) and the non-tapered antenna cavity with $\mu_{\mathrm{z}}=1$ (red) 


\section{Best Case Realized Gain for Tapered and Non-tapered Cavity}

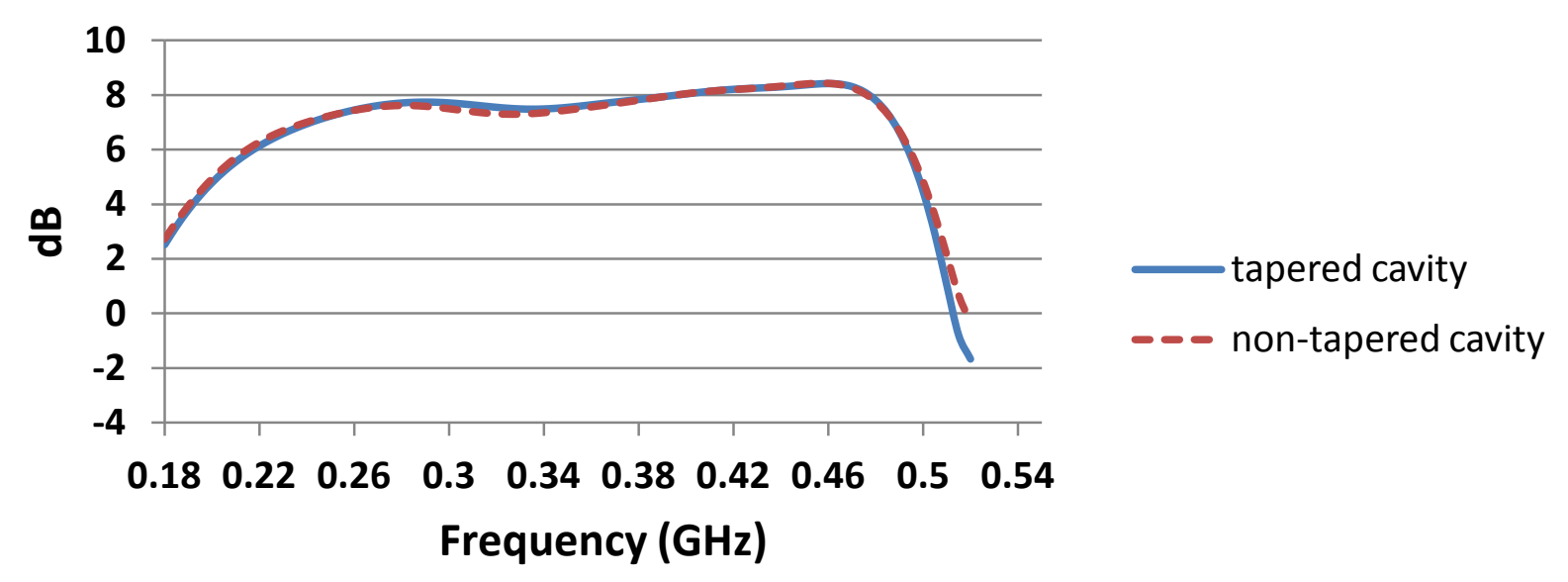

Fig. 11 Realized gain for the tapered antenna cavity with $\mu_{\mathrm{z}}=15$ (blue) and the non-tapered antenna cavity with $\mu_{z}=1$ (red)

\subsection{Cascaded Antenna with Feed Structure}

The results of the symmetric-probe-fed antenna models from Sections 3.1 and 3.2 were driven using two separate ports that are $180^{\circ}$ out of phase with an equal magnitude. This is an optimized way to drive the antenna, but in reality we would want a feed structure with a single input port and two output ports with $-3.0 \mathrm{~dB}$ insertion loss (this is a one-half power split) as well as a $180^{\circ}$ phase shift. The following subsections show the results for two different feed structures.

The following are the results for a commercial $180^{\circ}$ hybrid coupler and the non-tapered antenna of Fig. 8. The commercial splitter is the Werlatone 2-Way $180^{\circ}$ Combiner/Divider H7971-102. The pertinent S-parameter and phase difference information pertaining to this device is found in the Appendix.

Figure 12 shows how the two output ports 2 and 3 of the coupler connect to the two antenna input ports $1 \mathrm{~A}$ and $2 \mathrm{~A}$. All antenna dimensions are consistent with Table 2. 


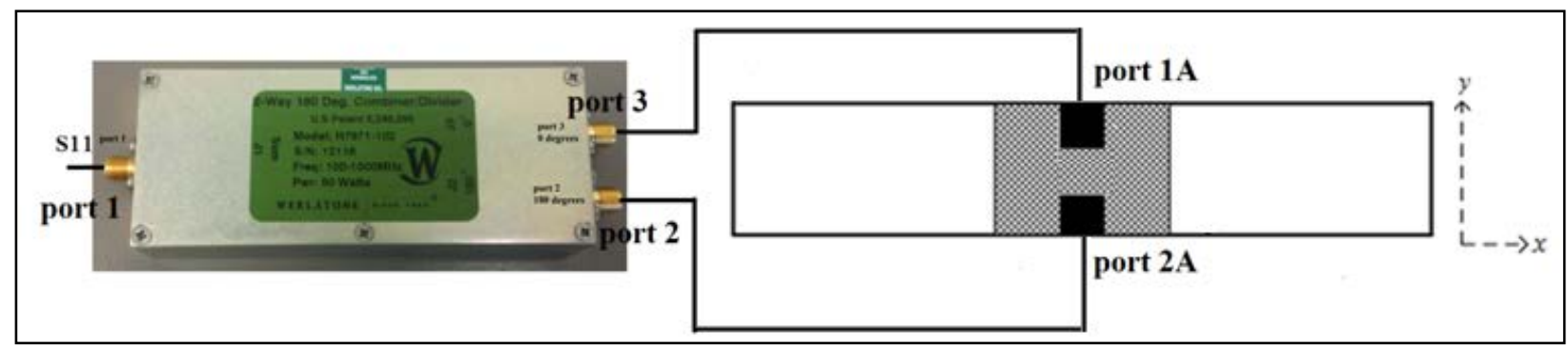

Fig. 12 The diagram showing the connectivity between the $180^{\circ}$ coupler and the two-port antenna

Figures 13-15 compare the performance of the system shown in Fig. 12 to those of the antennas shown in Fig. 8 to see if there is any improvement when using the commercial coupler. It should be noted that for the antenna with no coupler, the return loss is calculated at the input to port $1 \mathrm{~A}$ in Fig. 12, and for the antenna with the coupler, the return loss is calculated at the input to port 1. There is better than a 4-dB improvement in S11 due to the presence of the coupler and up to 0.75-dB degradation in realized gain due to the added insertion loss in S21 and S31 of the coupler.

Adding a commercial $180^{\circ}$ hybrid coupler has improved the return loss at the input to the system and increased the bandwidth with very little degradation in the realized gain. The antenna system now has better than a 2:1 VSWR from 220-505 MHz and better than a 3:1 VSWR from 200-515 MHz. The return loss of the antenna design was good enough that the $0.75-\mathrm{dB}$ degradation in the return loss is due almost solely to the insertion loss of the coupler and not due to any mismatch between the output port of the coupler and the input port of the antenna.

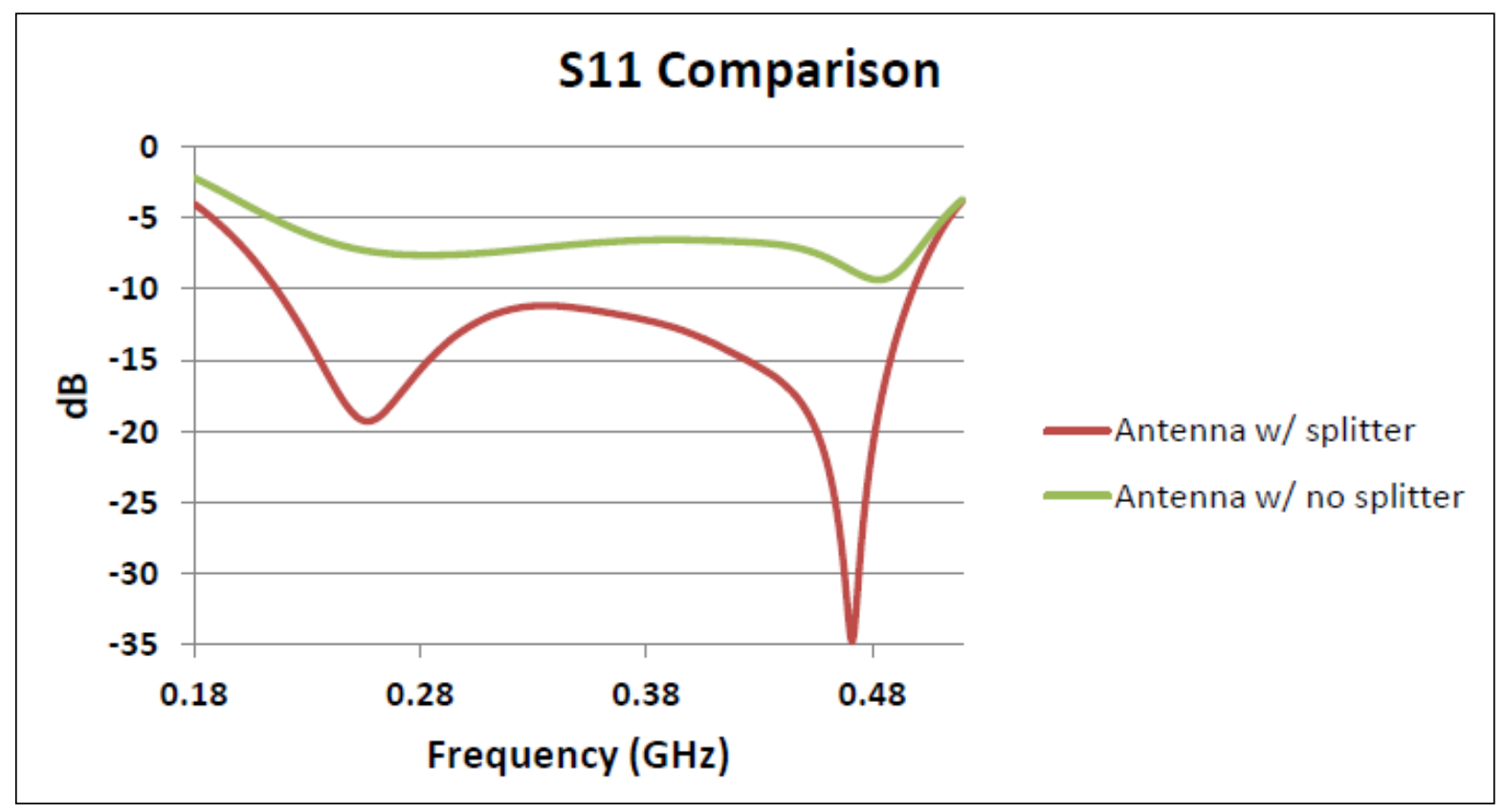

Fig. 13 Return loss vs. frequency at for the antenna of Fig. 12 (red) and Fig. 8 (green) 


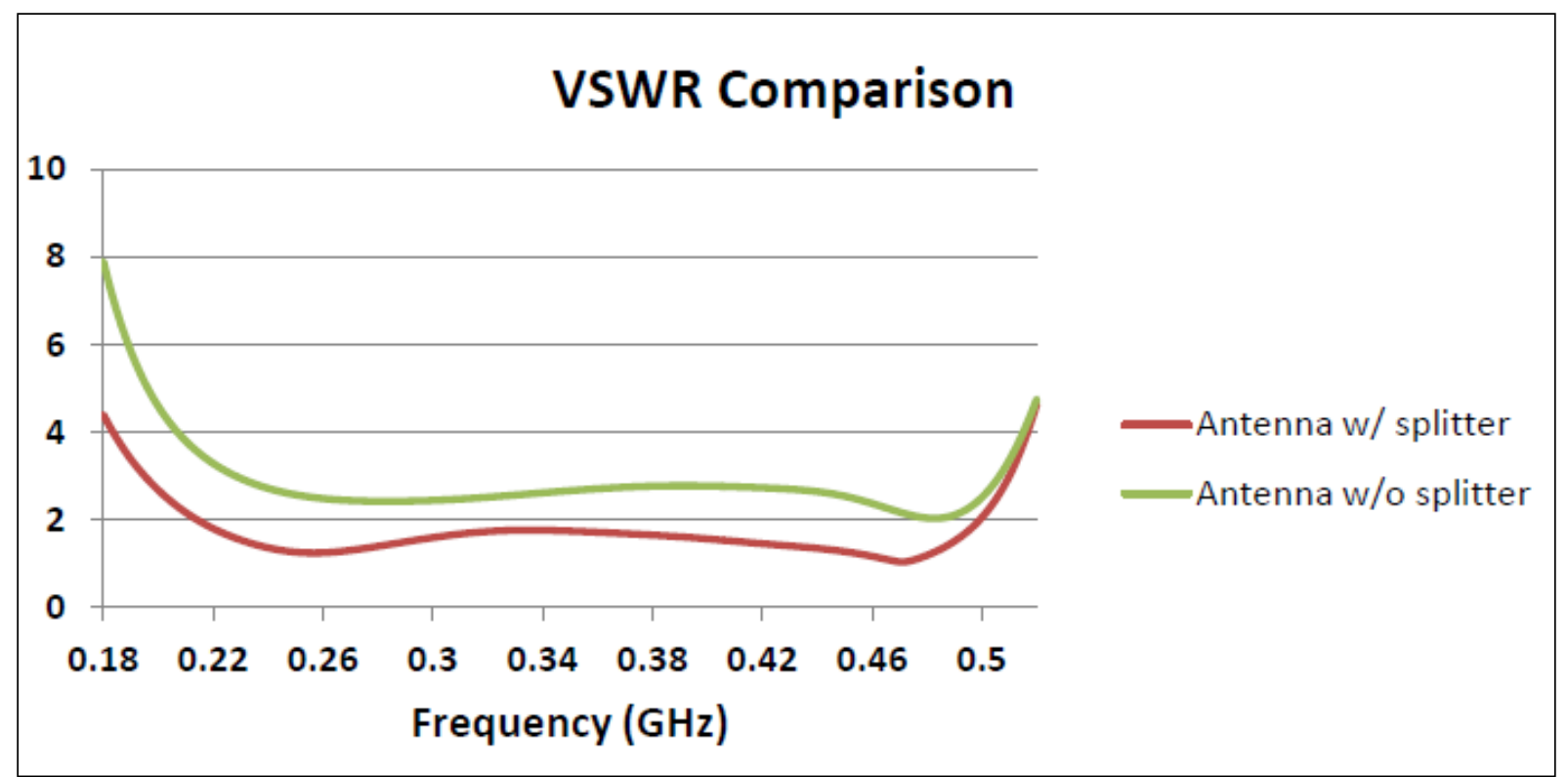

Fig. 14 VSWR vs. frequency plots for the antenna of Fig 12 (red) and Fig. 4 (green).

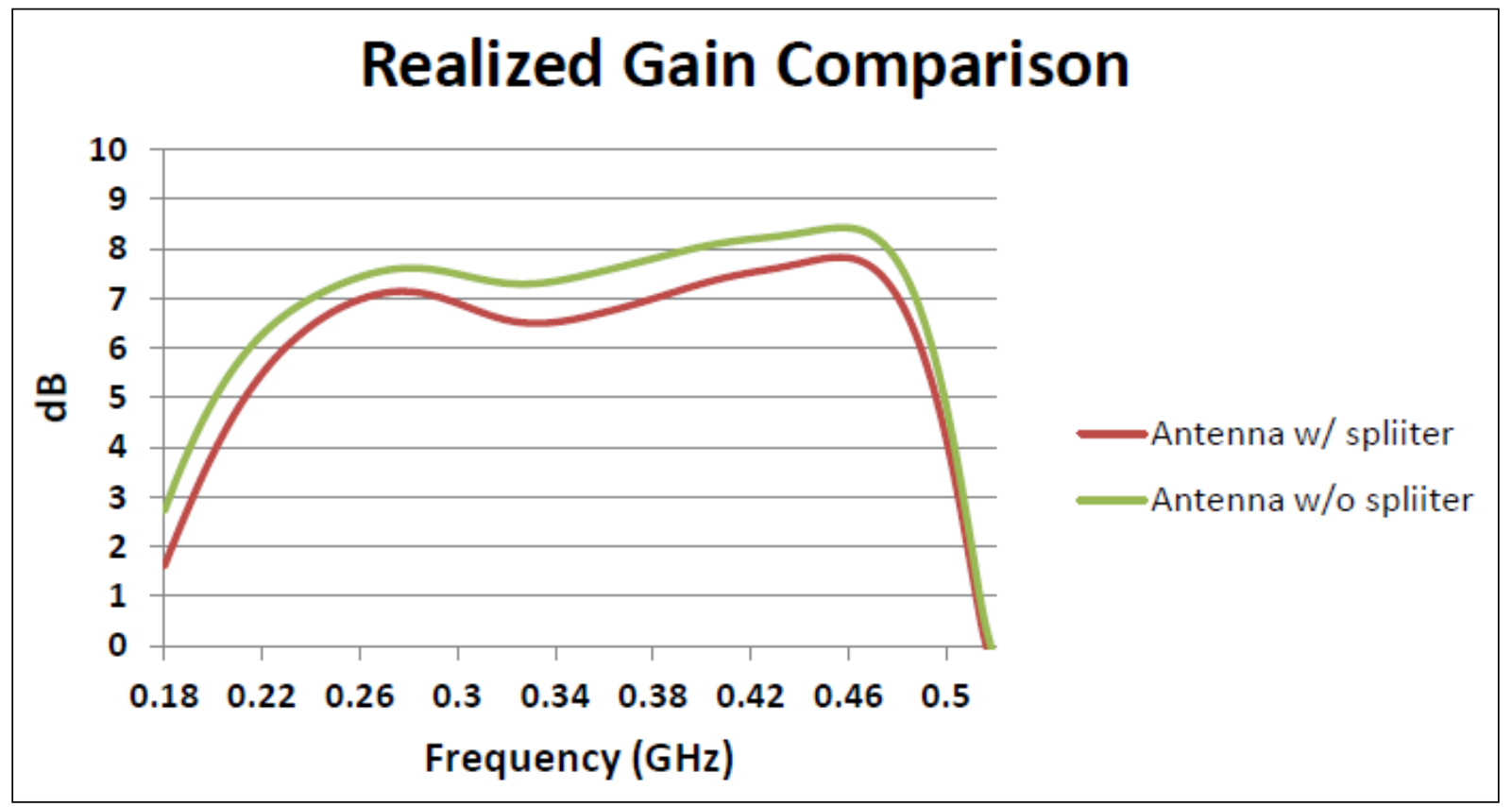

Fig. 15 Realized gain vs. frequency plots for the antenna of Fig 12 (red) and Fig. 4 (green) 


\section{Conclusions}

This report details the derivation of a LPA based on an anisotropic resonance condition of a partially loaded rectangular cavity. Results show that the shape of the cavity taper can be changed as needed with no degradation to the overall antenna performance through control of the permeability in the normal direction $\left(\mu_{z}\right)$. Research in this area has yielded a LPA design that has a profile of $d=0.055 \lambda_{o}\left(\lambda_{o} / 19\right)$ at $200 \mathrm{MHz}$ or $d=3.3$ inches with $\mu_{x}=1$. This antenna design has more than an octave of bandwidth from 200-515 MHz. This is a 78\% reduction in antenna profile compared to the traditional $\lambda_{o} / 4$ separation between radiating element and ground plane. The design has a positive realized gain from 180-515 MHz, a 3:1 VSWR from 200-515 MHz, and a 2:1 VSWR from 220-505 MHz. The 3:1 VSWR BW exceeds the design goals of 200-500 MHz.

Further investigation into matching techniques for larger values of $\mu_{x}$ is needed to try and reduce the profile of the antenna further. 


\section{References}

1. Lagarkov AN, Semeneko VN, Kisel VN, Christyaev VA. Development and Simulation of Microwave Artificial Magnetic Composites Utilizing Non-magnetic Inclusion. 2003.

2. Maslovski S, Ikonen P, Kolmakov I, Tretyakov S. Artificial Magnetic Materials Based on the New Magnetic Particle Metasolenoid. Progress in Electromagnetics Research (PIER). 2005;54:61-81.

3. Mitchell G. Comparison of Anisotropic versus Isotropic Metamaterials in Low Profile UHF Antenna Design; Adelphi (MD): US Army Research Laboratory (US); 2104. Report No.: ARL-TR-7012.

4. Meng F-Y, Wu Q, Fu Jai-H. Miniaturized Rectangular Cavity Resonator Based on Anisotropic Metamaterials Bilayer. Microwave and Optical Technology Letters. August 2008;50(8).

5. Meng F-Y, Wu Q, Li L-W. Transmission characteristics of wave modes in a rectangular waveguide filled with anisotropic metamaterial. Applied Physics A: Material Science. 2009;94:747-753. 


\section{Appendix. Werlatone $180^{\circ}$ Divider/Combiner}

This appendix shows the pertinent S-parameters of the $180^{\circ}$ hybrid coupler discussed in Section 3.3. This is in actuality a four-port device, but the fourth port is terminated inside the metal box.

Figure A-1 shows a picture of the device. There is one input port and two output ports with a $180^{\circ}$ phase difference. Its operational frequency range is $100-1000 \mathrm{GHz}$, but we are interested mostly in the $200-500 \mathrm{MHz}$ range. Figure A-2 shows the return loss at the input port of the coupler as measured on a network analyzer. Here we see a better than $-20 \mathrm{~dB}$ return loss across the entire frequency range of interest. Figure A-3 shows the insertion loss at the output ports of the coupler as measured on a network analyzer. There is a $0.25-\mathrm{dB}$ difference between the two outputs. These plots show a $0.55-0.75 \mathrm{~dB}$ difference in insertion loss from a perfect $-3.0 \mathrm{~dB}$ split. Figure A-4 shows the phase difference at the output ports of the coupler as measured on a network analyzer. This device maintains a phase separation of $180.5^{\circ}$ across frequency.

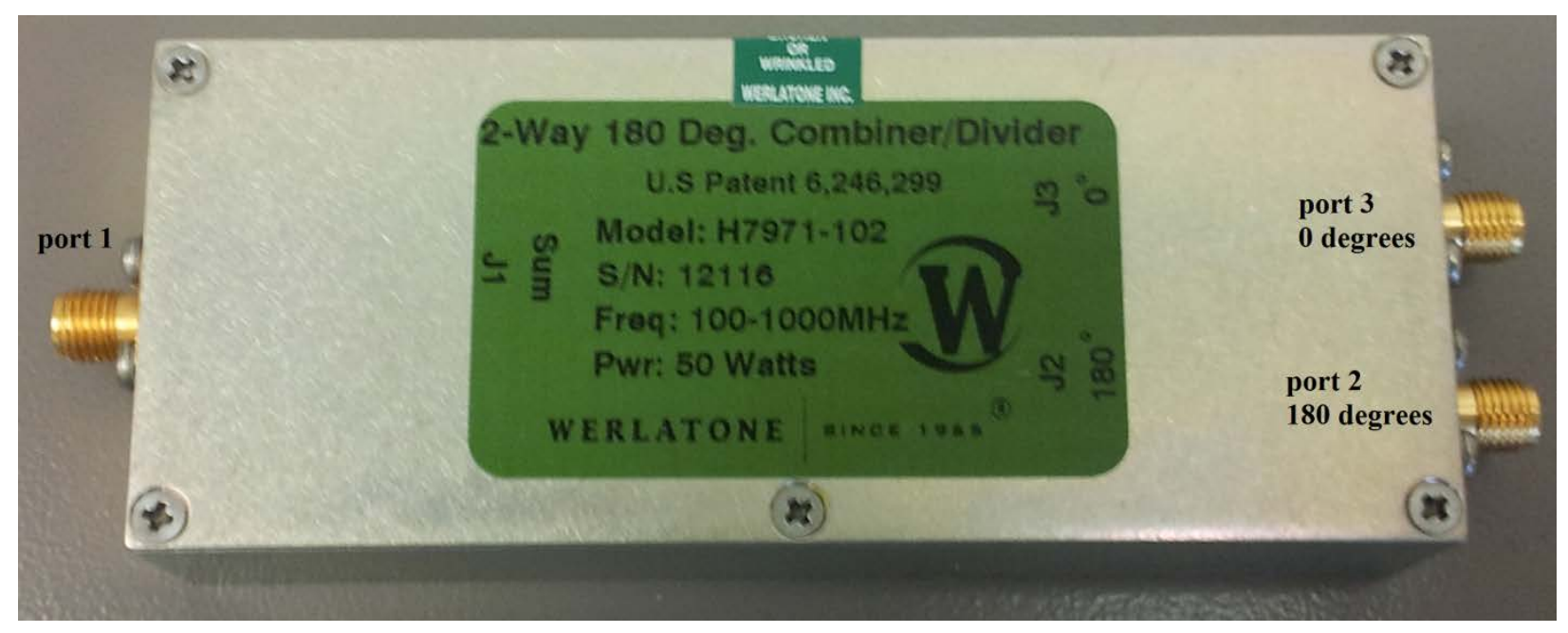

Fig. A-1 Picture of the $180^{\circ}$ coupler with the ports labeled. Port 1 is the input and ports 2 and 3 are the outputs. 


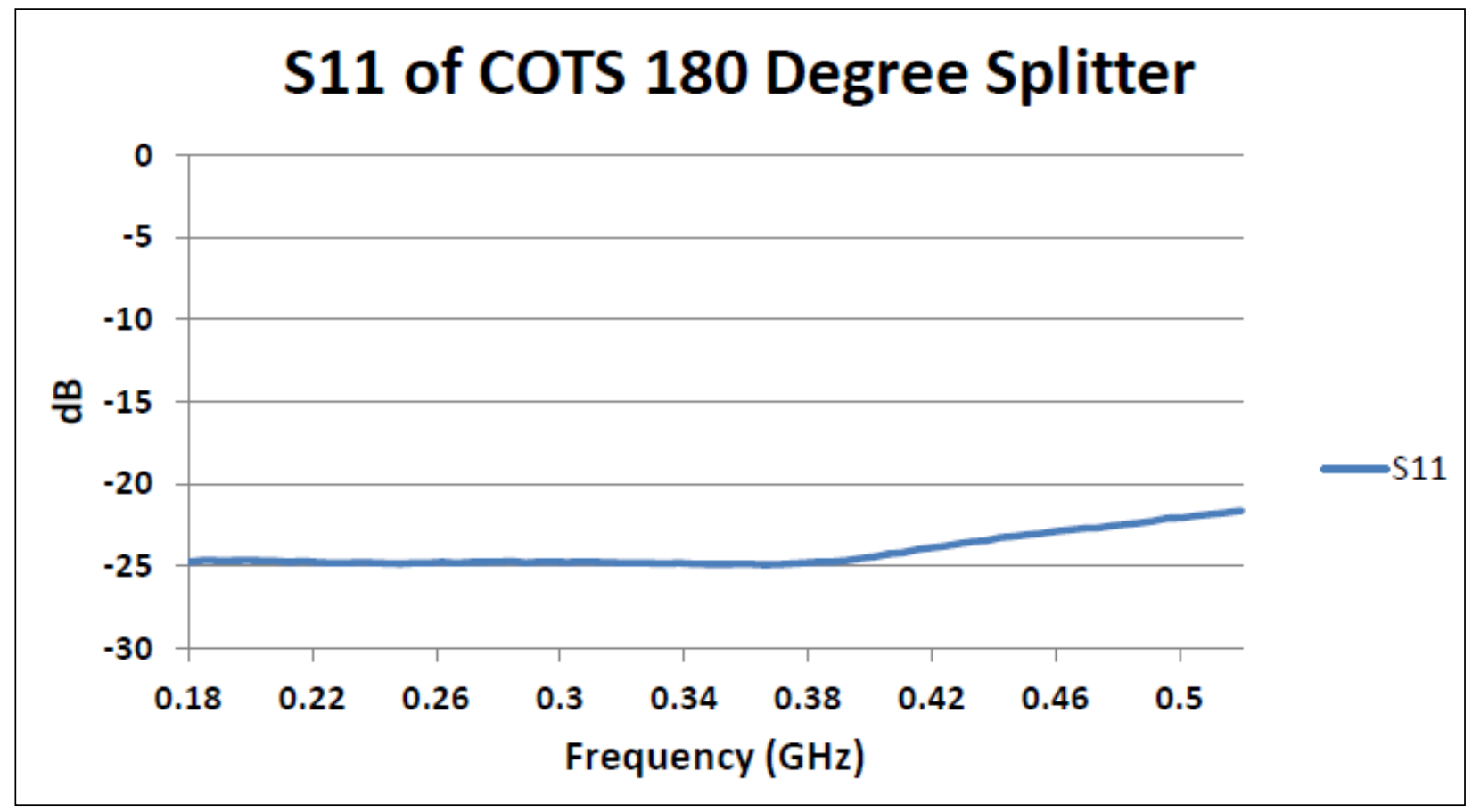

Fig. A-2 S11 of the Werlatone $180^{\circ}$ hybrid coupler

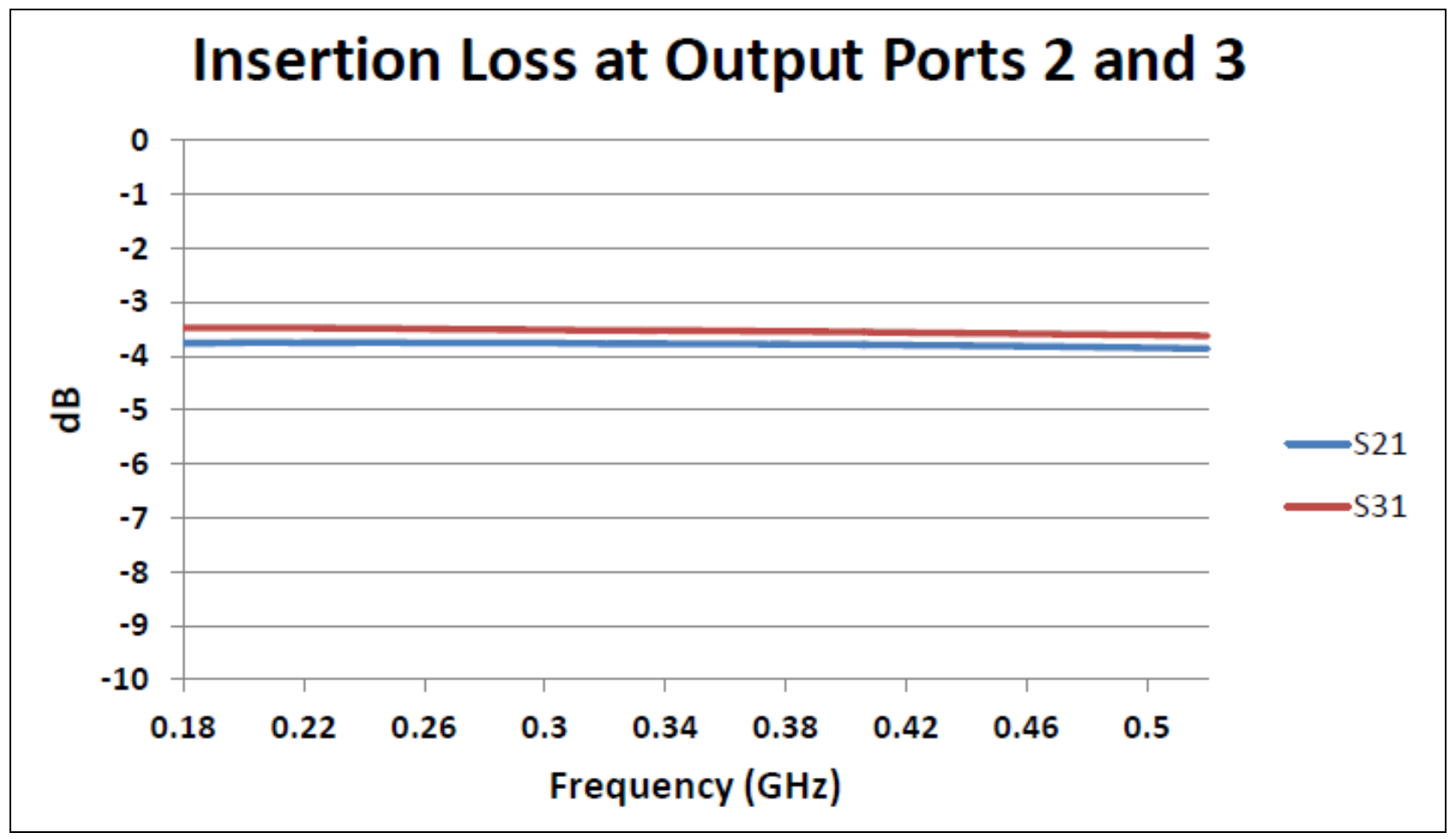

Fig. A-3 S21 and S31 of the Werlatone $180^{\circ}$ hybrid coupler 


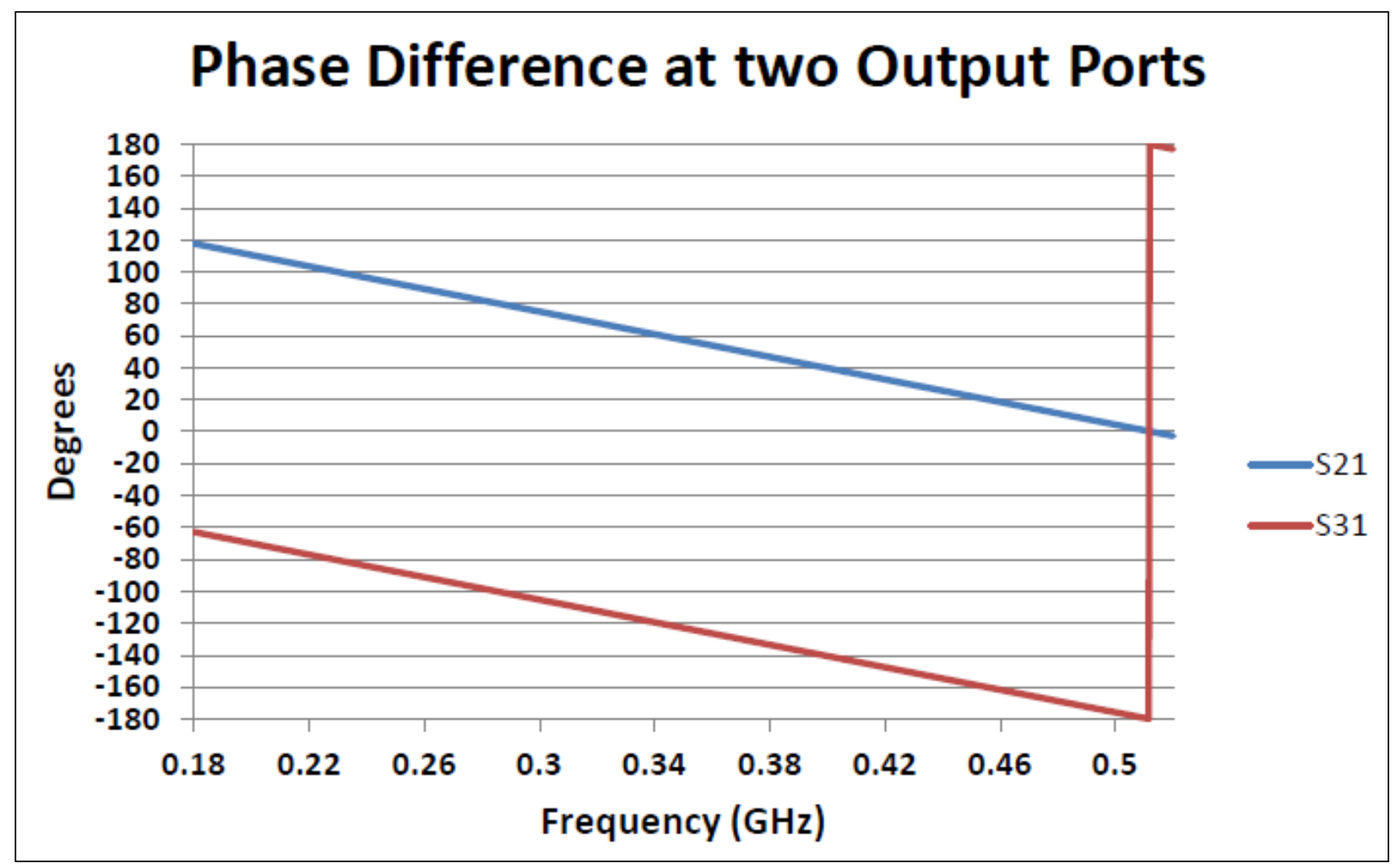

Fig. A-4 Phase difference at ports 2 and 3 of the $180^{\circ}$ coupler 


\author{
1 DEFENSE TECH INFO CTR \\ (PDF)ATTN DTIC OCA (PDF) \\ 2 US ARMY RSRCH LABORATORY \\ (PDF) ATTN IMAL HRA MAIL \& RECORDS MGMT \\ ATTN RDRL CIO LL TECHL LIB \\ 1 GOVT PRNTG OFC \\ (PDF) ATTN A MALHOTRA \\ 6 US ARMY RSRCH LAB \\ (PDF) ATTN RDRL SER M A ZAGHLOUL \\ ATTN RDRL SER M E ADLER \\ ATTN RDRL SER M G MITCHELL \\ ATTN RDRL SER M S WEISS \\ ATTN RDRL SER M T ANTHONY \\ ATTN RDRL SER M W O COBURN
}

